\title{
Dufour Effect on Transient MHD Double Convection Flow of Fractionalized Second-Grade Fluid with Caputo-Fabrizio Derivative
}

\author{
Imran Siddique, ${ }^{1}$ Sehrish Ayaz, ${ }^{1}$ and Fahd Jarad $\mathbb{D}^{2,3}$ \\ ${ }^{1}$ Department of Mathematics, University of Management and Technology, Lahore 54770, Pakistan \\ ${ }^{2}$ Department of Mathematics, Cankaya University, Etimesgut, Ankara, Turkey \\ ${ }^{3}$ Department of Medical Research, China Medical University Hospital, China Medical University, Taichung, Taiwan
}

Correspondence should be addressed to Fahd Jarad; fahd@cankaya.edu.tr

Received 3 May 2021; Accepted 25 August 2021; Published 11 September 2021

Academic Editor: Marcus Aguiar

Copyright (c) 2021 Imran Siddique et al. This is an open access article distributed under the Creative Commons Attribution License, which permits unrestricted use, distribution, and reproduction in any medium, provided the original work is properly cited.

\begin{abstract}
This article presents the problem, in which we study the unsteady double convection flow of a magnetohydrodynamics (MHD) differential-type fluid flow in the presence of heat source, Newtonian heating, and Dufour effect over an infinite vertical plate with fractional mass diffusion and thermal transports. The constitutive equations for the mass flux and thermal flux are modeled for noninteger-order derivative Caputo-Fabrizio (CF) with nonsingular kernel, respectively. The Laplace transform and Laplace inversion numerical algorithms are used to derive the analytical and semianalytical solutions for the dimensionless concentration, temperature, and velocity fields. Expressions for the skin friction and rates of heat and mass transfer from the plate to fluid with noninteger and integer orders, respectively, are also determined. Furthermore, the influence of flow parameters and fractional parameters $\alpha$ and $\beta$ on the concentration, temperature, and velocity fields are tabularly and graphically underlined and discussed. Furthermore, a comparison between second-grade and viscous fluids for noninteger and integer is also depicted. It is observed that integer-order fluids have greater velocities than noninteger-order fluids. This shows how the fractional parameters affect the fluid flow.
\end{abstract}

\section{Introduction}

The interest in fluid mechanics is truly significant within the sight of transport phenomena, which is a critical element in thermal, chemical, and mechanical engineering science. A few actual systems exist which can be utilized to move thermal energy and compound species through a phase and across limits of the phase. The three mechanisms for heat transfer are diffusion, convection, and radiation. The classification of convection of heat transfer into three consequent branches are natural (free), forced, and mixed convection, which is essential for the physical system that takes up the motion of the fluid. Free convection flows ensuing from the heat and mass transfer directed by the combined buoyancy effects because of temperature and concentration variations have been widely studied due to their applications in geotechnical engineering and chemical and bioengineering and in industrial activities [1]. Usually, the mass transfer due to the concentration disparity influences the rate of heat transfer. The driving force for the free convection is buoyancy, so its effects cannot be neglected whether the velocity of the fluid is small and change in temperature between the ambient fluid and surface is large enough [2-4].

Electrically conducting fluids also have accepted enough consideration from the researchers due to their extensive applications in industrial appliances. The MHD has its own practical implication, such as the tumor treating fields and power generation and earthquake assumption [5]. Parvin and Nasrin [6] have presented the analysis of the flow and 
heat transfer characteristics for MHD-free convection in inclusion with heated difficulties. They showed that the influence of the magnetic parameter on streamlines and isotherms is significant.

The energy flux which is due to a composition gradient is said to be the Dufour or diffusion-thermo effect. Such influences are significant when density differences occur in the flow regime. Such as when species are introduced at a surface in the fluid domain with different (lower) density than the surrounding fluid, Dufour effects can be beneficial. Also, when heat and mass transfers take place simultaneously in a moving fluid, it has a relationship between the fluxes and the driving potentials are of more twisting nature. It has been analyzed that an energy flux can be generated not only by temperature gradients but also by composition gradients as well. The diffusion-thermo effect was found to be of a considerable magnitude such that it cannot be negligible [7]. Dufour effects are essential in geothermal energy, hydrology, and nuclear waste disposal. In view of the importance of the diffusion-thermo effect, Kafoussias and Williams [8] studied the effect of thermal diffusion and diffusion-thermo on the mixed free forced convective and mass transfer boundary layer flow with temperature-dependent viscosity. Babu et al. [9] studied the diffusion-thermo and radiation effects on MHD-free convective heat and mass transfer flow past an infinite vertical plate in the presence of a chemical reaction of the first order. The dimensionless governing equations were solved with the Laplace transform technique. Rajput and Gupta [10] investigated the diffusion-thermo effect on unsteady free convection MHD flow past an exponentially accelerated plate through porous media with variable temperature and constant mass diffusion in an inclined magnetic field. Sharma and Buragohain [11] examined the Soret and Dufour effects on unsteady flow past an oscillating vertical plate with the help of numerical technique. Postelnicu [12] studied simultaneous heat and mass transfer by natural convection from a vertical plate embedded in an electrically conducting fluid saturated porous medium in the presence of Soret and Dufour effects using the Darcy-Boussinesq model. Gaikwad et al. [13] investigated the onset of double diffusive convection in two component couple of the stress fluid layer with Soret and Dufour effects using both linear and nonlinear stability analysis. Prakash et al. [14] considered the Dufour effects on unsteady MHD natural convection flow past a spontaneously started infinite vertical plate with variable temperature and constant mass diffusion through a permeable medium, and the dimensionless governing equations were solved in a closed form by utilizing the Laplace transform technique. They tracked down that the Dufour impact has critical effect on the velocity and temperature fields.

Numerous fluids in practical developments show nonNewtonian behavior because the consistent Newtonian fluids do not explicitly clarify the attributes of real fluids. Among non-Newtonian fluids, second-grade fluid is one of the viscoelastic fluids which were introduced by Rivlin [15] and Rivlin and Erickson [16]. Beard and Walters [17] are considered the pioneer of viscoelastic fluids. They developed the boundary layer theory for the second-grade fluids. This

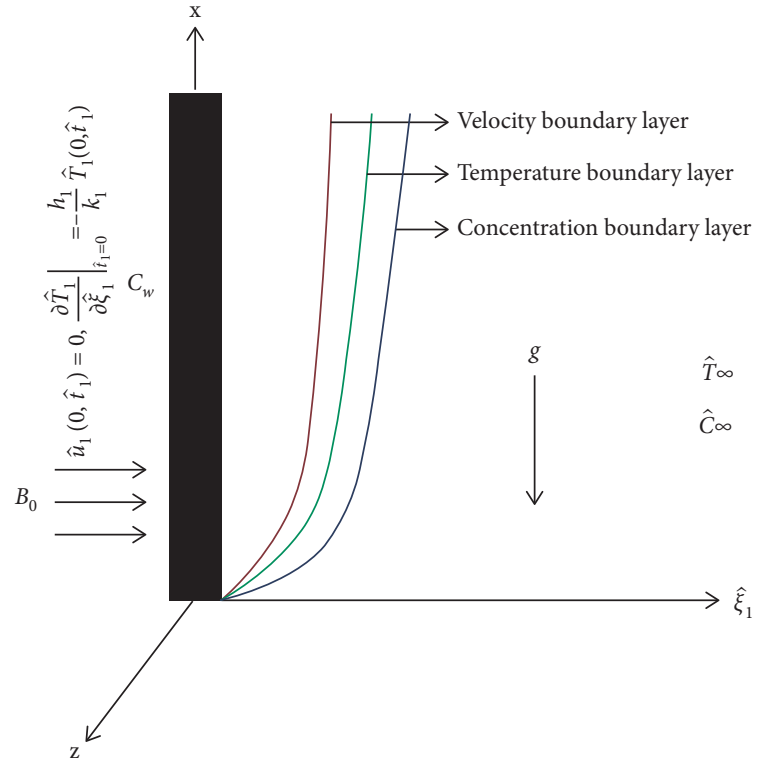

FIgURe 1: Flow geometry.

boundary layer theory for the second-grade fluids has motivated many researchers to really explore this kind of fluids with various conditions. Ariel [18] attained an interpretive solution for an incompressible laminar secondgrade fluid between the plates. Kecebas and Yurusoy [19] analyzed an unsteady two-dimensional power law fluid of the second grade and used a finite difference approach to solve reduced governing equations. Raftari [20] considered the MHD steady flow and heat transfer of a second-grade fluid and obtained an analytical solution. Aman et al. [21] analyzed the unsteady heat and mass transfer in secondgrade fluid over a flat plate with wall suction and injection.

Recently, it has progressively been seen as a dynamic tool through which a beneficial generalization of physical ideas can be obtained. Most fractional derivatives used are the Riemann-Liouville (RL) fractional derivative and the Caputo fractional derivative [22, 23]. It is observed that these operators exhibit obstacle in applications, such as the RL derivative of a constant is not zero, and the Laplace transform of the RL derivative involves terms which have no physical signification. The Caputo fractional derivative has excluded these difficulties, but the kernel of the definition is a singular function. Caputo and Fabrizio have introduced recently a new definition of the fractional derivatives with an exponential kernel without singularities [24]. The results that are been analyzed using these operators are expressed in complicated forms involving some generalized functions [25-34].

The innovation of the present paper is to examine the double convection flow of an incompressible differentialtype fluid near a vertical plate with heat source, Newtonian heating, and diffusion-thermo effect. Fractional derivative $\mathrm{CF}$ with nonsingular kernel is used in the constitutive equations of the mass flux and thermal flux to describe the diffusion and thermal processes, respectively. Semianalytical solutions of the dimensionless problems are established by 
virtue of the Laplace inversion numerical algorithm Gaver-Stehfest $[35,36]$. Expressions of skin friction, Sherwood and Nusselt numbers with fractional, and ordinary cases, respectively, are also determined. The results which we attained here are new and can be applied to other viscoelastic fluids. Applications of this research would be helpful in magnetic material processing and chemical engineering systems. At the end, the influence of flow parameters and the fractional parameter on the temperature and concentration field as well as on the velocity field are tabularly and graphically analyzed.

\section{Mathematical Model}

Let us consider the double convection flow of an electrically conducting incompressible differential-type fluid lying over an infinite vertical plate occupying in the $x \bar{\xi}_{1}$-plane with Newtonian heating as shown in Figure 1. Initially, the fluid and the plate are at rest and its temperature is $M$ (ambient fluid temperature) and the concentration level on the plate is $L^{-1}\left\{1 /\left(s^{2}+b_{1} s+b_{2}\right)\right\}=\left(2 / \sqrt{b_{1}^{2}-4 b_{2}}\right) \sinh \left(\left(\sqrt{b_{1}^{2}-4 b_{2}} / 2\right)\right.$ t) $e^{-\left(b_{1} / 2\right) t}$ (ambient fluid concentration). After time $T_{1}=0^{+}$, the heat transfer from the plate to the fluid is proportional to the local surface temperature $T_{1}$, and the concentration level on the plate is $\widehat{C}_{w}$ (wall concentration) which is thereafter kept constant. Presume that the influence of double convection and viscous dissipation in momentum and energy equations are insignificant, respectively. Also, the direction of flow has no pressure gradient. We assume that the velocity, temperature, and concentration are functions of $\bar{\xi}_{1}$ and $\bar{t}_{1}$ only. For such a flow, the constraint of incompressibility is identically satisfied. Taking the consistent Boussinesq approximation, the convection flow is governed by the following set of partial differential equations $[32,33,37]$ :

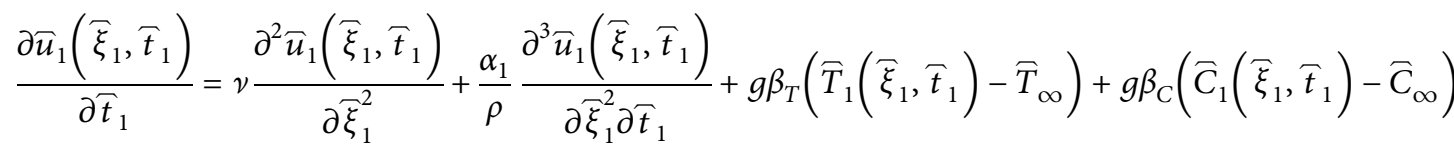

$$
\begin{aligned}
& -\frac{\sigma B_{0}^{2} \tilde{u}_{1}\left(\tilde{\xi}_{1}, \tau_{1}\right)}{\rho}, \quad \bar{\xi}_{1}, T_{1}>0
\end{aligned}
$$

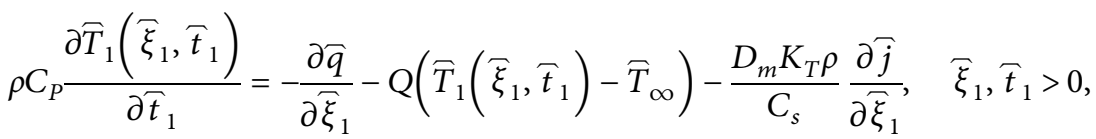

$$
\begin{aligned}
& \bar{q}=-k_{1} \frac{\partial \widehat{T}_{1}}{\partial \bar{\xi}_{1}}
\end{aligned}
$$

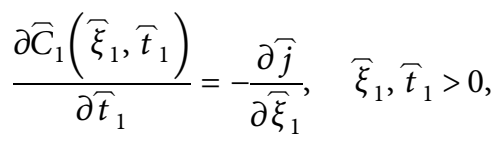

$$
\begin{aligned}
& \overparen{j}=-D_{m} \frac{\partial \bar{C}_{1}}{\partial \bar{\xi}_{1}} .
\end{aligned}
$$

The appropriate initial and boundary conditions are as follows: 


$$
\begin{aligned}
& \overparen{u}_{1}\left(\widetilde{\xi}_{1}, 0\right)=0 \\
& \widehat{T}_{1}\left(\bar{\xi}_{1}, 0\right)=\overparen{T}_{\infty} \\
& \overparen{C}_{1}\left(\bar{\xi}_{1}, 0\right)=\overparen{C}_{\infty}, \quad \overparen{\xi}_{1} \geq 0 \\
& \widetilde{u}_{1}\left(0, T_{1}\right)=0 \text {, } \\
& \left.\frac{\partial \widehat{T}_{1}}{\partial \widetilde{\xi}_{1}}\right|_{\bar{\xi}_{1}=0}=-\frac{h_{1}}{k_{1}} T_{1}\left(0, T_{1}\right) \text {, } \\
& \overparen{C}_{1}\left(0, T_{1}\right)=\overparen{C}_{w}, \quad T_{1}>0, \\
& \overparen{u}_{1}\left(\bar{\xi}_{1}, \overparen{t}_{1}\right) \longrightarrow 0 \\
& \overparen{T}_{1}\left(\bar{\xi}_{1}, \widetilde{t}_{1}\right) \longrightarrow \overparen{T}_{\infty}, \\
& \bar{C}_{1}\left(\widetilde{\xi}_{1}, \widetilde{t}_{1}\right) \longrightarrow \widetilde{C}_{\infty}, \quad \bar{\xi}_{1} \longrightarrow 0, \widetilde{t}_{1}>0 .
\end{aligned}
$$

On introducing the nondimensional quantities from Appendix A into equations (1)-(8), we get the following nondimensional partial differential equations:

$$
\begin{aligned}
\frac{\partial u}{\partial t}= & \frac{\partial^{2} u(y, t)}{\partial y^{2}}+\gamma \frac{\partial^{3} u(y, t)}{\partial y^{2} \partial t}+\operatorname{Gr} \theta(y, t) \\
& +\operatorname{GmC}(y, t)-\operatorname{Mu}(y, t), \quad y, t>0, \\
\operatorname{Pr} \frac{\partial \theta}{\partial t}= & -\frac{\partial q}{\partial y}-S \theta(y, t)-\operatorname{PrDu} \frac{\partial j}{\partial y}, \quad y, t>0, \\
q= & -\frac{\partial \theta}{\partial y}, \\
\frac{\partial C}{\partial t}= & -\frac{\partial j}{\partial y}, \quad y, t>0, \\
j= & -\frac{1}{\operatorname{Sc}} \frac{\partial C}{\partial y},
\end{aligned}
$$

with the initial and boundary conditions

$$
\begin{aligned}
& u(y, 0)=0, \\
& \theta(y, 0)=0, \\
& C(y, 0)=0, \quad y \geq 0, \\
& u(0, t)=0, \\
& \left.\frac{\partial \theta}{\partial y}\right|_{y=0}=-[\theta(0, t)+1], \\
& C(0, t)=1,
\end{aligned}
$$

$$
\begin{aligned}
& u(y, t) \longrightarrow 0, \\
& \theta(y, t) \longrightarrow 0, \\
& C(y, t) \longrightarrow 0, \quad \text { as } y \longrightarrow \infty, t>0 .
\end{aligned}
$$

To establish a model with time-fractional derivatives, we assume a thermal process with memory illustrated by the next generalized fractional constitutive equation for thermal flux and mass diffusion, respectively $[38,39]$ :

$$
\begin{aligned}
& j(y, t)=-\frac{1}{\mathrm{Sc}}{ }^{\mathrm{CF}} D_{t}^{\alpha} \frac{\partial C(y, t)}{\partial y}, \quad \alpha \in[0,1), \\
& q(y, t)=-{ }^{\mathrm{CF}} D_{t}^{\beta} \frac{\partial \theta(y, t)}{\partial y}, \quad \beta \in[0,1),
\end{aligned}
$$

where the CF time-fractional derivative ${ }^{\mathrm{CF}} D_{t}^{p}(\cdot)$ of order $p$ is defined by [24]

$$
{ }^{\mathrm{CF}} D_{t}^{p} u(y, t)=\frac{1}{1-p} \int_{0}^{t} \exp \left(\frac{-p(t-\tau)}{1-p}\right) \frac{\partial u(y, \tau)}{\partial \tau} \mathrm{d} \tau, \quad p \in[0,1) .
$$
follows:

The Laplace transform of the CF time derivative is as

$$
L\left\{{ }^{\mathrm{CF}} D_{t}^{p} u(y, t)\right\}=\frac{s L\{u(y, t)\}-u(y, 0)}{(1-p) s+p} .
$$

Remark 1. If $u(y, 0)=0$ and $p \longrightarrow 0$, equation (20) becomes $L\left\{{ }^{\mathrm{CF}} D_{t}^{p} u(y, t)\right\}=L\{u(y, t)\}$. In this case, the generalized mass flux and thermal flux equations (17) and (18) reduce to the classical Fourier's law and Fick's law equations (11) and (13), respectively. For $p \longrightarrow 1$,

$$
\lim _{p \longrightarrow 1} L\left\{{ }^{\mathrm{CF}} D_{t}^{p} u(y, t)\right\}=s L\{u(y, t)\}-u(y, 0)=L\left\{\frac{\partial u(y, t)}{\partial t}\right\} .
$$

For the correspondence, it will be as follows:

$$
\frac{\partial u(y, t)}{\partial t}=\lim _{p \longrightarrow 1}{ }^{\mathrm{CF}} D_{t}^{p} u(y, t)
$$

\section{Solution of the Problem}

3.1. Concentration Field. Applying the Laplace transform to equations (12), (13), third equation in (15), third equation in (16), and (17), keep in mind the initial condition (third equation in (14)), and after simplification, we obtain the transformed problem:

$$
\begin{gathered}
\frac{\partial^{2} \bar{C}(y, s)}{\partial y^{2}}-\operatorname{Sc}[(1-\alpha) s+\alpha] \bar{C}(y, s)=0, \quad y>0 \\
\bar{C}(0, s)=\frac{1}{s}, \\
\bar{C}(y, s) \longrightarrow 0, \quad \text { as } y \longrightarrow \infty
\end{gathered}
$$


The differential equation (24) gives the solution with respect to condition (24):

$$
\bar{C}(y, s)=\frac{1}{s} e^{-y a_{0} \sqrt{s+a}},
$$

where $a_{0}=\sqrt{\operatorname{Sc}(1-\alpha)}$ and $a=(a / 1-\alpha)$.

The inverse Laplace transform of the above equation is perceived using equation (A.3) from Appendix C.

$$
C(y, t)=\frac{1}{2}\left[e^{y a_{0} \sqrt{a}} \operatorname{erfc}\left(\frac{y a_{0}}{2 \sqrt{t}}+\sqrt{a t}\right)+e^{-y a_{0} \sqrt{a}} \operatorname{erfc}\left(\frac{y a_{0}}{2 \sqrt{t}}-\sqrt{a t}\right)\right] .
$$

The local mass transfer coefficient from the plate to the fluid, that is, Sherwood number, is taken by the subsequent relation:

$$
\mathrm{Sh}_{g}=j(0, t)=-\frac{1}{(1-\alpha) \mathrm{Sc}} L^{-1}\left\{\left.\frac{s}{s+a} \frac{\partial \bar{C}(y, s)}{\partial y}\right|_{y=0}\right\}=\frac{a_{0}}{(1-\alpha) \mathrm{Sc}}\left\{\frac{e^{-a t}}{\sqrt{\pi t}}\right\} .
$$

The obtained results in equations (26) and (27) identical results exist in [29] (equations (3.9) and (3.22)).

3.2. Concentration Field for an Ordinary Case $(\alpha \longrightarrow 0)$. In special case when $\alpha \longrightarrow 0$, we obtain the ordinary concentration field by means of the equation (A.4) from Appendix C.

$$
C(y, t)=\operatorname{erfc}\left(\frac{y a_{1}}{2 \sqrt{t}}\right) .
$$

Similarly, we obtain the expression for the Sherwood number as follows:

$$
\mathrm{Sh}_{o}=\sqrt{\frac{\mathrm{Sc}}{\pi t}}
$$

\section{Temperature Field}

Applying the Laplace transform to equations (10), (11), second equation in (15), second equation in (16), and (18), using the initial condition (second equation in (14)), after simplification, we get the transformed problem:

$$
\begin{gathered}
\frac{\partial^{2} \bar{\theta}}{\partial y^{2}}-\frac{b_{0}\left(s^{2}+b_{1} s+b_{2}\right)}{s} \bar{\theta}(y, s)=\frac{b_{3}(s+b)}{s} e^{-y a_{0} \sqrt{s+a}}, \\
\left.\frac{\partial \bar{\theta}(y, s)}{\partial y}\right|_{y=0}=-\left[\bar{\theta}(0, s)+\frac{1}{s}\right] \\
\bar{\theta}(y, s) \longrightarrow 0, \quad \text { as } y \longrightarrow \infty
\end{gathered}
$$

Differential equation (30) gives the following solution with the boundary condition (31):

$$
\begin{aligned}
\bar{\theta}(y, s)= & \frac{1}{s} \frac{1}{\sqrt{b_{0}}} \frac{1}{\sqrt{w_{1}(s)}+\xi} e^{-y \sqrt{b_{0}} \sqrt{w_{1}(s)}} \\
& +\frac{s b_{6}}{w_{2}(s)}\left\{\frac{e^{-y a_{0} \sqrt{s+a}}}{s}+\frac{1}{s} \frac{1}{\sqrt{b_{0}}} \frac{e^{-y \sqrt{b_{0}} \sqrt{w_{1}(s)}}}{\sqrt{w_{1}(s)}+\xi}\left(1-a_{0} b_{6} \sqrt{s+a}\right)\right\},
\end{aligned}
$$

where $b_{i}, i=0, \ldots, 6$ and $\xi$ are constants given in Appendix B 


$$
\begin{aligned}
b & =\frac{\beta}{1-\beta}, \\
w_{1}(s) & =\frac{b_{0}\left(s^{2}+b_{1} s+b_{2}\right)}{s}, \\
w_{2}(s) & =\frac{s^{2}+b_{4} s+b_{5}}{s+b} .
\end{aligned}
$$

To find the inverse Laplace transform, equation (34) can be written as follows:

$$
\bar{\theta}(y, s)=\bar{\theta}_{1}(s) \cdot \bar{\theta}_{2}(y, s)+\bar{\theta}_{3}(s) \cdot\left[\bar{\theta}_{4}(y, s)+\bar{\theta}_{6}(s) \cdot \bar{\theta}_{5}(y, s)\right],
$$

where

$$
\begin{aligned}
\bar{\theta}_{1}(s) & =\frac{1}{s} \frac{1}{\sqrt{b_{0}}} \frac{1}{\sqrt{w_{1}(s)}+\xi} \\
\bar{\theta}_{2}(y, s) & =e^{-y \sqrt{b_{0}} \sqrt{w_{1}(s)}}, \\
\bar{\theta}_{3}(y, s) & =\frac{s b_{6}}{w_{2}(s)},
\end{aligned}
$$

$$
\begin{aligned}
\bar{\theta}_{4}(y, s) & =\frac{e^{-y a_{0} \sqrt{s+a}}}{s} \\
\bar{\theta}_{5}(s) & =\frac{1}{s} \frac{1}{\sqrt{b_{0}}} \frac{e^{-y \sqrt{b_{0}} \sqrt{w_{1}(s)}}}{\sqrt{w_{1}(s)}+\xi} \\
\bar{\theta}_{6}(y, s) & =1-a_{0} b_{6} \sqrt{s+a} .
\end{aligned}
$$

The inverse Laplace transform of equation (35) is obtained using (A.3), (A.5)-(A.11) from Appendix C, and by taking convolution theorem, we will get the following equation:

$$
\theta(y, t)=\theta_{1}(t) * \theta_{2}(y, t)+\theta_{3}(t) *\left[\theta_{4}(y, t)+\theta_{6}(t) * \theta_{5}(y, t)\right],
$$

where $*$ denotes the convolution product.

$$
\begin{aligned}
\theta_{1}(t)= & -\frac{1}{\sqrt{b_{0}}}-\frac{1}{b_{0}} \int_{0}^{t} G_{2}(\tau) \mathrm{d} \tau \\
\theta_{2}(y, t)= & e^{-b_{1} t} \frac{e^{-\left(b_{0} y^{2} / 4 t\right)}}{\sqrt{\pi t}}-\int_{0}^{\infty} \frac{e^{-b_{1} u-\left(b_{0} y^{2} / 4 u\right)}}{\sqrt{\pi u}} \sqrt{\frac{b_{2} u}{t-u}} J_{1}\left(2 \sqrt{b_{2} u(t-u)}\right) \mathrm{d} u \\
\theta_{3}(t)= & \delta(t)+\frac{2\left(b-b_{4}\right)}{\sqrt{b_{4}^{2}-4 b_{5}}} \int_{0}^{\infty} \delta^{\prime}(t-\tau) e^{-\left(b_{4} / 2\right) \tau} \sinh \left(\frac{\sqrt{b_{4}^{2}-4 b_{5}}}{2} \tau\right) \mathrm{d} \tau \\
& -\frac{2 b_{5}}{\sqrt{b_{4}^{2}-4 b_{5}}} \sinh \left(\frac{\sqrt{b_{4}^{2}-4 b_{5}}}{2} t\right) e^{-\left(b_{4} / 2\right) \tau}, \\
\theta_{4}(y, t)= & \text { equation }(27), \\
\theta_{5}(y, t)= & \theta_{1}(t) * \theta_{2}(y, t)=\int_{0}^{t} \theta_{1}(t-\tau) \theta_{2}(y, \tau) \mathrm{d} \tau \\
\theta_{6}(t)= & \delta(t)+a_{0} b_{6} \frac{e^{-a t}}{2 t \sqrt{\pi t}},
\end{aligned}
$$

here

$$
G_{2}(t)=e^{-b_{1} t}\left(\frac{1}{\sqrt{\pi t}}-\xi e^{\xi^{2} t} \operatorname{erf} c(\xi \sqrt{t})\right)-\int_{0}^{\infty} e^{-b_{1} u}\left(\frac{1}{\sqrt{\pi u}}-\xi e^{\xi^{2} u} \operatorname{erf} c(\xi \sqrt{u})\right) \sqrt{\frac{b_{2} u}{t-u}} J_{1}\left(2 \sqrt{b_{2} u(t-u)}\right) \mathrm{d} u
$$


The local coefficient of heat transfer from the plate to the fluid, in terms of the Nusselt number, is as follows:

$$
\begin{aligned}
\mathrm{Nu}_{g} & =q(0, t)=-\frac{1}{1-\beta} L^{-1}\left\{\left.\frac{\partial \bar{\theta}(y, s)}{\partial y}\right|_{y=0}\right\} \\
& =\frac{1}{1-\beta} L^{-1}\left\{\frac{\sqrt{w_{1}(s)}}{(s+b)\left(\sqrt{w_{1}(s)}+\xi\right)}\left(1+\frac{b_{6}}{w_{2}(s)}\left(1+a_{0} b_{6} \sqrt{s+a}\right)\right)+\frac{a_{0} b_{6}}{w_{2}(s) \sqrt{s+a}}\right\} .
\end{aligned}
$$

The inverse Laplace transform of equation (39) is established numerically and is described in Section 6 in the tabular form.
4.1. Temperature Field for an Ordinary Case $(\alpha \longrightarrow 0, \beta \longrightarrow 0)$. In special case, that is, $\alpha \longrightarrow 0, \beta \longrightarrow 0$, to obtain the ordinary temperature field by means of equations (A.10) and (A.11) from Appendix C,

$$
\begin{aligned}
\theta(y, t)= & \frac{1}{\sqrt{\operatorname{Pr}}} \int_{0}^{t} \frac{1}{\sqrt{\pi \tau}} e^{-a_{1} \tau-\left(\operatorname{Pr} y^{2} / 4 \tau\right)}-\varsigma e^{\sqrt{\operatorname{Pr}} y+\left(\varsigma^{2}-a_{1}\right) \tau} \operatorname{erfc}\left(\frac{\sqrt{\operatorname{Pr}} y}{2 \sqrt{\tau}}+\varsigma \sqrt{\tau}\right) \mathrm{d} \tau \\
& +\frac{a_{2}}{2} \int_{0}^{t}\left[e^{-a_{3} \tau-i y \sqrt{S c} \sqrt{a_{3}}} \operatorname{erfc}\left(\frac{y \sqrt{\operatorname{Sc}}}{2 \sqrt{\tau}}-i \sqrt{a_{3} \tau}\right)+e^{-a_{3} \tau+i y \sqrt{S c} \sqrt{a_{3}}} \operatorname{erfc}\left(\frac{y \sqrt{\mathrm{Sc}}}{2 \sqrt{\tau}}+i \sqrt{a_{3} \tau}\right)\right] \mathrm{d} \tau,
\end{aligned}
$$

where $a_{i}, i=1, \ldots, 3$ and $\zeta$ are constants given in Appendix B.

$$
\begin{aligned}
\mathrm{Nu}_{o}= & 1-\frac{\varsigma}{a_{1}-\varsigma^{2}}\left[\sqrt{a_{1}} \operatorname{erf}\left(\sqrt{a_{1} t}\right)-\left(e^{-\left(a_{1}-\xi^{2}\right) t} \operatorname{erfc}(\varsigma \sqrt{t})-1\right)\right] \\
& +a_{2} \sqrt{\mathrm{Sc}} \int_{0}^{\infty} \frac{1}{\sqrt{\pi u}}\left(\delta(t-u)-a_{3} e^{-a_{3}(t-u)}\right) \mathrm{d} u .
\end{aligned}
$$

\section{Velocity Field}

Applying the Laplace transform to equation (9), with the initial condition (first equation in (14)) and using the expressions of equations (25) and (32), we obtain the following Laplace transform velocity:

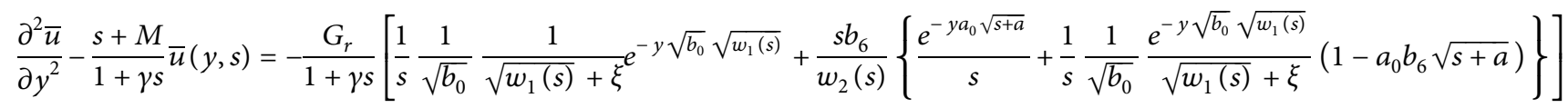

$$
\begin{aligned}
& -\frac{G_{m}}{1+\gamma s} \frac{1}{s} e^{-y a_{0} \sqrt{s+a}}
\end{aligned}
$$

Here, $\bar{u}(y, s)$ represents the function of the Laplace transform and $u(y, t)$ that has to satisfy the conditions.

$$
\begin{aligned}
& \bar{u}(0, s)=0, \\
& \bar{u}(y, s) \longrightarrow 0, \quad \text { as } y \longrightarrow \infty .
\end{aligned}
$$


The ordinary differential equation (42) gives the solution with subject to conditions (43):

$$
\begin{aligned}
\bar{u}(y, s)= & \frac{1}{s^{3}+d_{0} s^{2}+d_{1} s+d_{2}} \cdot \frac{1}{\sqrt{w_{1}(s)}+\xi}\left\{A_{1}+A_{2} \frac{s}{w_{2}(s)}\right\}\left[\frac{1}{s} e^{-y c_{0} \sqrt{(s+M) /\left(s+d_{3}\right)}}-e^{-y \sqrt{b_{0} w_{1}(s)}}\right] \\
& +\frac{A_{2}}{s^{3}+d_{0} s^{2}+d_{1} s+d_{2}} \cdot \frac{s \sqrt{s+a}}{\sqrt{w_{1}(s)}+\xi} \cdot \frac{a_{0}}{w_{2}(s)}\left[\frac{1}{s} e^{-y c_{0} \sqrt{(s+M) /\left(s+d_{3}\right)}}+e^{-y \sqrt{b_{0} w_{1}(s)}}\right] \\
& +\frac{1}{s^{2}+a_{4} s+a_{5}}\left\{A_{3}+A_{4} \frac{1}{w_{2}(s)}\right\}\left[\frac{1}{s} e^{-y c_{0} \sqrt{(s+M) /\left(s+d_{3}\right)}}-e^{-y a_{0} \sqrt{s+a}}\right],
\end{aligned}
$$

where $\quad c_{0}=1 / \sqrt{\gamma} \quad a_{i}, i=4,5, \quad A_{j}, j=0, \ldots, 4 \quad$ and $d_{k}, k=0, \ldots, 3$ are all constants given in Appendix B.
The skin friction coefficient corresponding to this motion is as follows:

$$
\begin{aligned}
\tau_{g}= & \frac{\partial u(0, t)}{\partial y}=L^{-1}\left\{\frac{\partial \bar{u}(0, s)}{\partial y}\right\} \\
= & L^{-1}\left[\frac{1}{s^{3}+d_{0} s^{2}+d_{1} s+d_{2}} \cdot \frac{1}{\sqrt{w_{1}(s)}+\xi}\left\{A_{1}+A_{2} \cdot \frac{s}{w_{2}(s)}\right\}\left(\frac{c_{0}}{s} \sqrt{\frac{s+M}{s+d_{3}}}-\sqrt{b_{0} w_{1}(s)}\right)\right. \\
& +\frac{A_{2}}{s^{3}+d_{0} s^{2}+d_{1} s+d_{2}} \frac{s \sqrt{s+a}}{\sqrt{w_{1}(s)}+\xi} \cdot \frac{a_{0}}{w_{2}(s)}\left(\frac{c_{0}}{s} \sqrt{\frac{s+M}{s+d_{3}}}+\sqrt{b_{0} w_{1}(s)}\right) \\
& \left.+\frac{1}{s^{2}+a_{4} s+a_{5}} \cdot\left\{\frac{A_{3}}{w_{2}(s)}+A_{4}\right\}\left(\frac{c_{0}}{s} \sqrt{\frac{s+M}{s+d_{3}}}-a_{0} \sqrt{s+a}\right)\right] .
\end{aligned}
$$

The inverse Laplace transform of equations (44) and (45) will be found numerically in Section 6 by applying the Stehfest's algorithm [35].
In this special case, that is, $\gamma \longrightarrow 0$, we will obtain the velocity field for fractional viscous fluid from equation (44) as follows:

\subsection{Velocity Field for Fractional Viscous Fluid $(\gamma \longrightarrow 0)$.}

$$
\begin{aligned}
& \bar{u}_{2}(y, s)=\frac{1}{s^{2}+p_{1} s+p_{2}} \cdot \frac{1}{\sqrt{w_{1}(s)}+\xi}\left\{A_{5}+A_{6} \frac{s}{w_{2}(s)}\right\}\left[\frac{1}{s} e^{-y \sqrt{s+M}}-e^{-y \sqrt{b_{0} w_{1}(s)}}\right]+\frac{A_{6}}{s^{2}+p_{1} s+p_{2}} . \\
& \frac{s \sqrt{s+a}}{\sqrt{w_{1}(s)}+\xi} \cdot \frac{a_{0}}{w_{2}(s)}\left[\frac{1}{s} e^{--y \sqrt{s+M}}+e^{-y \sqrt{b_{0} w_{1}(s)}}\right]+\frac{1}{s+p_{3}}\left\{A_{7}+A_{8} \frac{1}{w_{2}(s)}\right\}\left[\frac{1}{s} e^{-y \sqrt{s+M}}-\frac{1}{s} e^{-y a_{0} \sqrt{s+a}}\right],
\end{aligned}
$$

where $A_{i}, i=5, \ldots, 8$ and $p_{j}, j=0, \ldots, 3$ are all constants given in Appendix B.

The Laplace transform of equation (46) is established numerically and described in Section 6.
5.2. Velocity Field for Ordinary Second-Grade Fluid $(\alpha \longrightarrow 0, \beta \longrightarrow 0)$. In this ordinary case, where $\alpha \longrightarrow 0$, $\beta \longrightarrow 0$, the expression of flow of velocity for the second-grade fluid given in equation (44) would be illustrated as follows: 


$$
\begin{aligned}
\bar{u}(y, s)= & \frac{A_{9}}{s^{2}+p_{4} s+p_{5}} \cdot \frac{1}{\sqrt{s+a_{1}}+\zeta^{\circ}}\left[\frac{1}{s} e^{-y c_{0} \sqrt{(s+M) /\left(s+d_{3}\right)}}-\frac{1}{s} e^{-y \sqrt{\operatorname{Pr}} \sqrt{s+a_{1}}}\right] \\
& +\frac{1}{s^{2}+p_{4} s+p_{5}}\left\{\frac{A_{10}}{s+a_{3}}+A_{11}\right\} \cdot\left[\frac{1}{s} e^{-y c_{0} \sqrt{(s+M) /\left(s+d_{3}\right)}}-\frac{1}{s} e^{-y \sqrt{S c} \sqrt{s}}\right],
\end{aligned}
$$

where $A_{i}, i=9, \ldots, 11$ and $p_{j}, j=4,5$ are constants given in Appendix B.

Equation (47) can be expressed as follows:

$$
\bar{u}(y, s)=\bar{u}_{1}(s) \cdot\left[\bar{u}_{2}(y, s)-\bar{u}_{3}(y, s)\right]+\bar{u}_{4}(s) \cdot\left[\bar{u}_{5}(y, s)-\bar{u}_{6}(y, s)\right] .
$$

The inverse Laplace transform of equation (48) using equations (A.3), (A.4), (A.7), (A.9), and (A.13) from

Appendix $\mathrm{C}$ as well as the convolution theorem is given as follows:

$$
u(y, t)=u_{1}(t) *\left[u_{2}(y, t)-u_{3}(y, t)\right]+u_{4}(t) *\left[u_{5}(y, t)-u_{6}(y, t)\right]
$$

where $*$ denotes the convolution product.

$$
\begin{aligned}
& u_{1}(t)=\frac{2 A_{9}}{\sqrt{p_{4}^{2}-4 p_{5}}} e^{-\left(p_{4} / 2\right) t} \int_{0}^{t} \sinh \left(\frac{\sqrt{p_{4}^{2}-4 p_{5}}}{2}(t-\tau)\right)\left\{\frac{1}{\sqrt{\pi \tau}}-\zeta e^{\zeta^{2} \tau} \operatorname{erf} c(\zeta \sqrt{\tau})\right\} e^{\left(\left(p_{4} / 2\right)-a_{1}\right) \tau} \mathrm{d} \tau, \\
& u_{2}(y, t)=e^{-y c_{0}}-\frac{y c_{0} \sqrt{M-d_{3}}}{2 \sqrt{\pi}} \int_{0}^{\infty} \int_{0}^{t} \frac{1}{\sqrt{t}} e^{-\left(d_{3} t-\left(\left(y c_{0}\right)^{2} / 4 \tau\right)-\tau\right)} I_{1}\left(2 \sqrt{\left(M-d_{3}\right) \tau t}\right) \mathrm{d} t \mathrm{~d} \tau, \\
& u_{3}(y, s)=\frac{1}{2}\left[e^{y \sqrt{\operatorname{Pr}} \sqrt{a_{1}}} \operatorname{erf} c\left(\frac{\sqrt{\operatorname{Pr}} y}{2 \sqrt{t}}+\sqrt{a_{1} t}\right)+e^{-y \sqrt{\operatorname{Pr}} \sqrt{a_{1}}} \operatorname{erfc}\left(\frac{\sqrt{\operatorname{Pr}} y}{2 \sqrt{t}}-\sqrt{a_{1} t}\right)\right], \\
& u_{4}(y, t)=\frac{2 A_{10}}{\sqrt{p_{4}^{2}-4 p_{5}}} e^{-\left(p_{4} / 2\right) t} \int_{0}^{t} \sinh \left(\frac{\sqrt{p_{4}^{2}-4 p_{5}}}{2}(t-\tau)\right) e^{\left(\left(p_{4} / 2\right)-a_{3}\right) \tau} \mathrm{d} \tau \\
& +A_{11} \frac{2}{\sqrt{p_{4}^{2}-4 p_{5}}} \sinh \left(\frac{\sqrt{p_{4}^{2}-4 p_{5}}}{2} t\right) e^{-\left(p_{4} / 2\right) t}, \\
& u_{5}(y, t)=u_{2}(y, t), \\
& u_{6}(y, t)=\operatorname{erfc}\left(\frac{y \sqrt{\mathrm{Sc}}}{2 \sqrt{t}}\right)
\end{aligned}
$$

5.3. Velocity Field for Ordinary Viscous Fluid $(\alpha \longrightarrow 0, \beta \longrightarrow 0, \gamma \longrightarrow 0)$. In this special case where $\alpha \longrightarrow 0, \beta \longrightarrow 0$, and $\gamma \longrightarrow 0$, the velocity expression for ordinary viscous given in equation (44), takes a form as follows:

$$
\begin{aligned}
u_{4}(y, s) & =\frac{A_{12}}{s+p_{6}} \frac{1}{\sqrt{s+a_{1}}+\zeta}\left[\frac{1}{s} e^{-y \sqrt{s+M}}-\frac{1}{s} e^{-y \sqrt{\operatorname{Pr}} \sqrt{s+a_{1}}}\right] \\
& +\frac{1}{s+p_{7}}\left\{\frac{A_{13}}{s+a_{3}}+A_{14}\right\}\left[\frac{1}{s} e^{-y \sqrt{s+M}}-\frac{1}{s} e^{-y \sqrt{S c} \sqrt{s}}\right],
\end{aligned}
$$


where $A_{i}, i=12, \ldots, 14$ and $p_{j}, j=6,7$ are all constants Equation (51) can be expressed as follows: given in Appendix $\mathrm{B}$.

$$
\bar{u}_{4}(y, s)=\bar{u}_{1}(s) \cdot\left[\bar{u}_{2}(y, s)-\bar{u}_{3}(y, s)\right]+\bar{u}_{4}(s) \cdot\left[\bar{u}_{5}(y, s)-\bar{u}_{6}(y, s)\right] .
$$

The inverse Laplace transform of equation (52), using (A.3), (A.4), and (A.9) from Appendix C, then by the convolution theorem, is given as follows:

$$
u(y, t)=u_{1}(t) *\left[u_{2}(y, t)-u_{3}(y, t)\right]+u_{4}(t) *\left[u_{5}(y, t)-u_{6}(y, t)\right]
$$

where

$$
\begin{aligned}
u_{1}(t) & =A_{12} e^{-p_{6} t} \int_{0}^{t} e^{\left(p_{6}-a_{1}\right) \tau}\left\{\frac{1}{\sqrt{\pi \tau}}-\zeta e^{\zeta^{2} \tau} \operatorname{erf} c(\zeta \sqrt{\tau})\right\} \mathrm{d} \tau, \\
u_{2}(y, t) & =\frac{1}{2}\left[e^{y p_{0} \sqrt{M}} \operatorname{erfc}\left(\frac{y p_{0}}{2 \sqrt{t}}+\sqrt{M t}\right)+e^{-y p_{0} \sqrt{M}} \operatorname{erfc}\left(\frac{y p_{0}}{2 \sqrt{t}}-\sqrt{M t}\right)\right], \\
u_{3}(y, t) & =\frac{1}{2}\left[e^{y \sqrt{\operatorname{Pr}} \sqrt{a_{1}}} \operatorname{erf} c\left(\frac{y \sqrt{\operatorname{Pr}}}{2 \sqrt{t}}+\sqrt{a_{1} t}\right)+e^{-y \sqrt{\operatorname{Pr}} \sqrt{a_{1}}} \operatorname{erf} c\left(\frac{y a_{2}}{2 \sqrt{t}}-\sqrt{a_{1} t}\right)\right], \\
u_{4}(t) & =A_{13}\left(\frac{e^{-p_{7} t}-e^{-a_{5} t}}{a_{3}-p_{7}}\right)+A_{14} e^{-a_{3} t}, \\
u_{5}(y, t) & =u_{2}(y, t), \\
u_{6}(y, t) & =\operatorname{erf} c\left(\frac{y \sqrt{\mathrm{Sc}}}{2 \sqrt{t}}\right) .
\end{aligned}
$$

\section{Numerical Results and Discussion}

The MHD second-grade fluid on an infinite vertical plate is considered with Newtonian heating, heat source, and diffusion-thermo effects. Time-fractional derivative $\mathrm{CF}$ with a nonsingular kernel is used in the constitutive equations of the mass flux and thermal flux to describe the diffusion and thermal processes, respectively. The magnetic field is introduced in the fluid flow which acts as opposing force to fluid motion. The expressions for dimensionless concentration, temperature, velocity fields, skin friction, and Sherwood and Nusselt numbers are obtained by means of the Laplace transform technique. Solutions for the classical model corresponding to the integer-order derivative are also obtained as limiting cases. All the parameters and profiles which are used here are dimensionless. It is observed initially in a consequence of the fractionalize parameters $\alpha$ and $\beta$ on the concentration, temperature, and velocity of fluid flow. Along with the effect of the absorption parameter $S$, the Prandtl number Pr, Dufour number Du, and time $t$ on the temperature as well as the impact of Schmidt number Sc, and Reynolds number Re on the concentration and fluid velocity are studied. The consequence of the thermal and mass Grashof number Gr and Gm second-grade parameter $\gamma$ and magnetic parameter $M$ for velocity is also presented.

Figures 2(a) and 3(a) present the dimensionless temperature and concentration profiles for distinct values of the fractional parameters $\alpha$ and $\beta$. As probable, the fluid temperature and concentration are decreasing functions with respect to their fractional parameters. Their values are maximum near the plate and smoothly decrease to zero for increasing $y$. Figure 4(a) was drawn to interpret the effect of the fractional parameters $\alpha$ and $\beta$ on the fluid velocity. If we give the same values to fractional parameters, the fluid flow velocity raises by increasing the values of $\alpha$ and $\beta$. The influence of the Schmidt number Sc on the fluid concentration is presented in Figure 2(b). It can be clearly seen from the figure that the concentration level of the fluid decreases whenever $S c$ is increasing. By the increase in time $t$, we observed from Figure 2(c) that the concentration profile is increasing. 


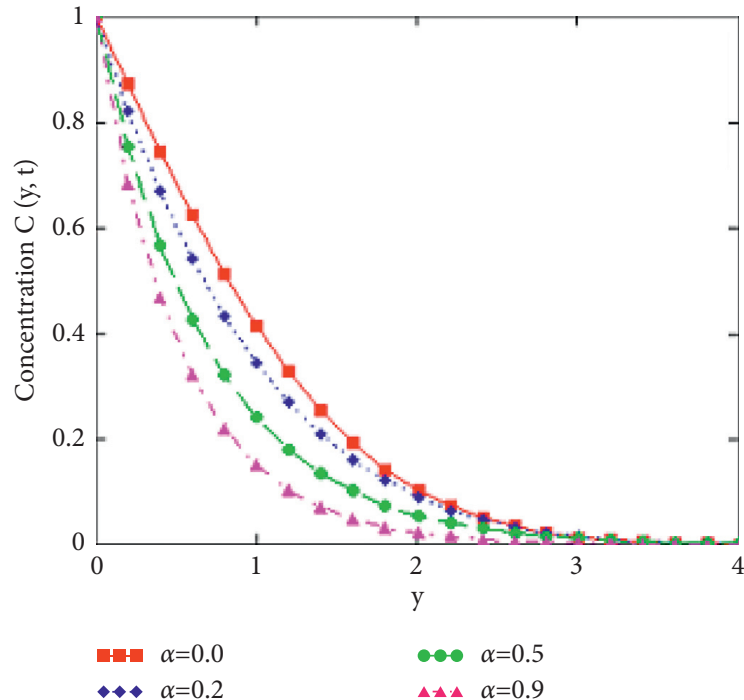

(a)

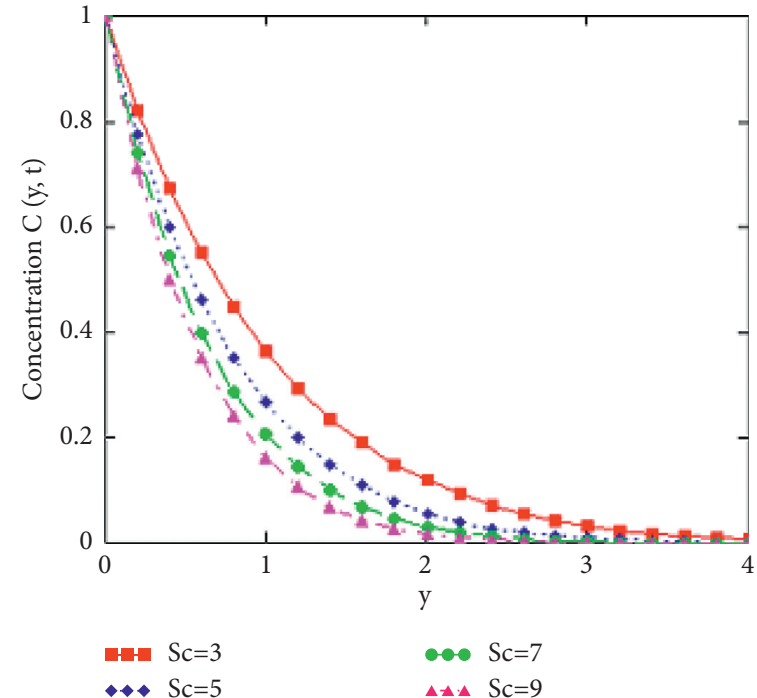

(b)

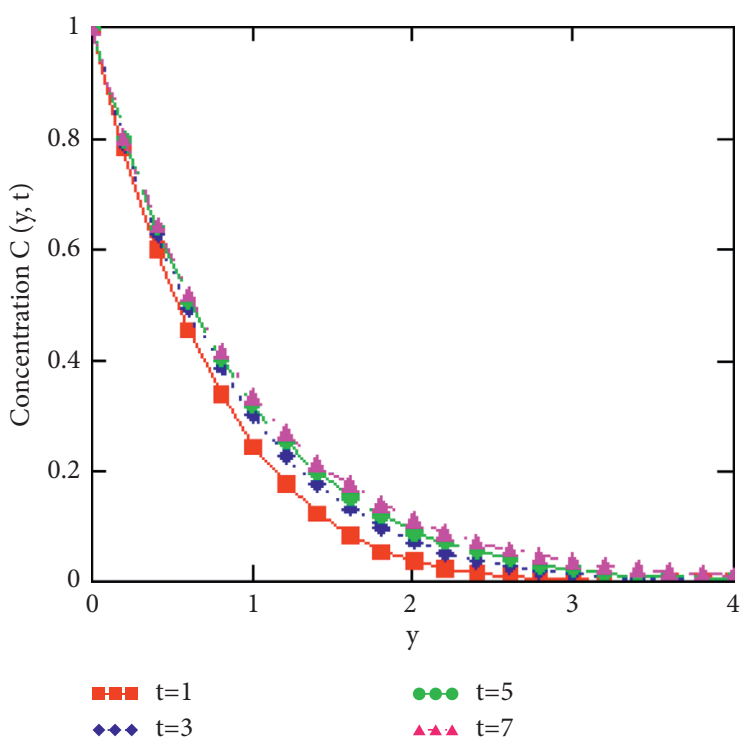

(c)

Figure 2: Concentration profiles vs. for $y$ at $\alpha=0.3, \mathrm{Sc}=4, t=3$.

The influence of the Prandtl number Prand absorption parameter $S$ is shown in Figures 3(b) and 3(c). It is noticed that by increasing the values of $\operatorname{Pr}$ and $S$, the temperature profile is decreasing while increasing corresponding to the values of the Schmidt number Sc as presented in Figure 3(d). Figures 3(e) and 4(e) illustrate the temperature and velocity profiles for different values of the Dufour number Du. It is observed that the thermal diffusivity as well as the velocity profile both are increasing and the boundary layer thickness gets maximized. In addition, by increasing in time $t$, the temperature profile and velocity profile both are increasing as shown in Figures 3(f) and 4(j).

It is seen in Figures 4(b) $-4(\mathrm{~d})$ that by assigning the higher values to $\operatorname{Pr}, S$, Sc, and $\gamma$, the fluid velocity profile decreases. The MHD principle is used for controlling the flow field in the essential direction by changing the making of the boundary layer. The variation of the velocity profile with different values of the magnetic parameter $M$ is shown in Figure 4(i). Increase in the values of $M$ shows the reduction in velocity. We agree with this result as expected that the magnetic field exerts a retarding effect on the mixed convection flow. Figure $4(\mathrm{~g})$ is plotted to see the impact of thermal Grashof Gr. Gr is the ratio of buoyancy forces to viscous forces on the motion of the fluid, which stimulates free or inner convection. It is found that the fluid flow velocity is increasing by increasing the values of Gr. Figure 4(f) is plotted to allocated the influence of the mass Grashof $\mathrm{Gm}$. It generates due to the change in concentration by a change in the density of a fluid, and it is the ratio of buoyancy forces to viscous forces; we found that by increasing $\mathrm{Gm}$, the velocity profile increases. 
12

Complexity

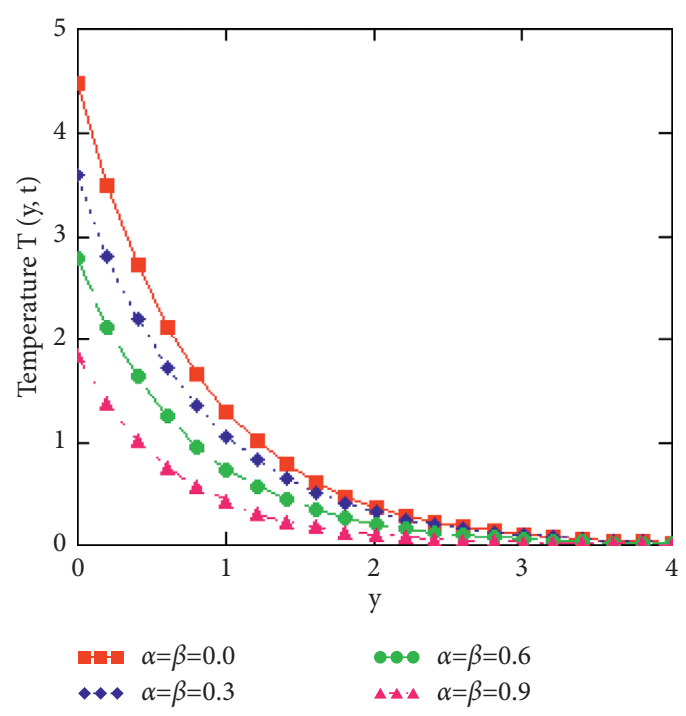

(a)

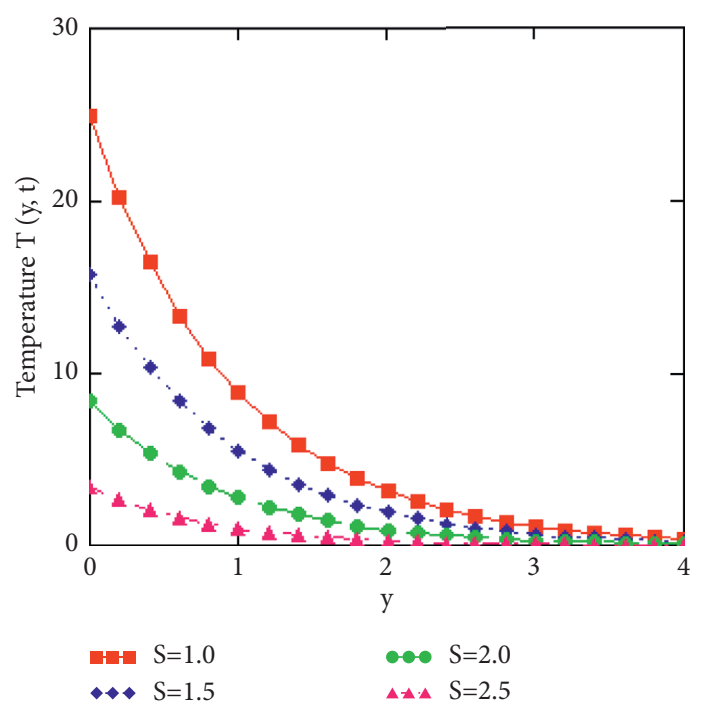

(c)

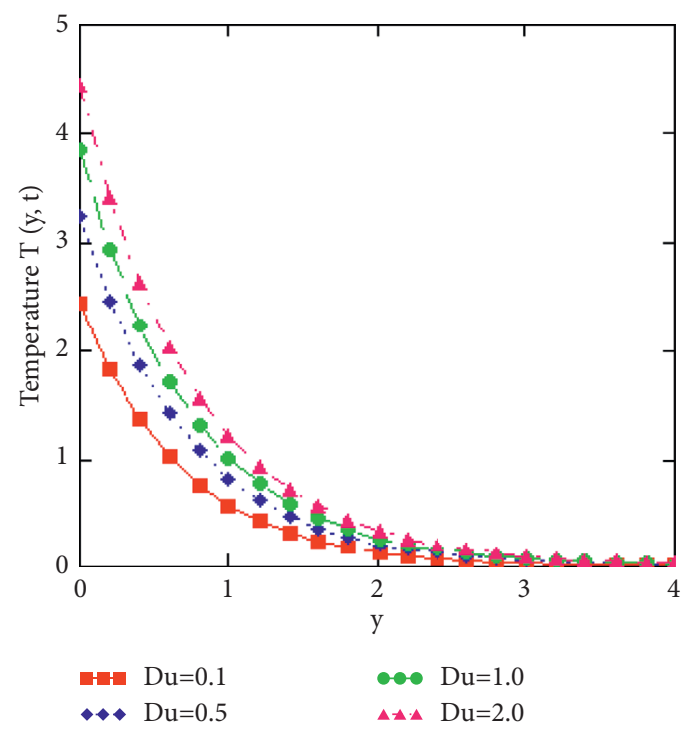

(e)

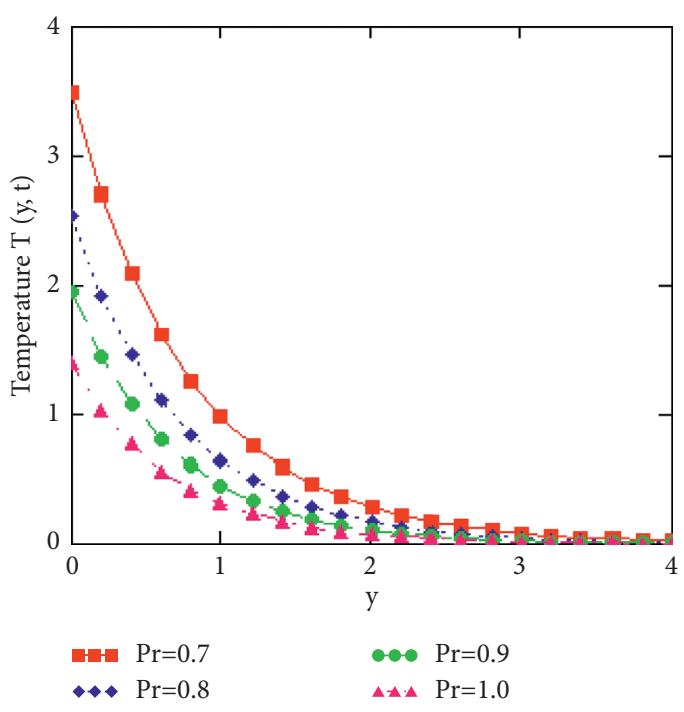

(b)

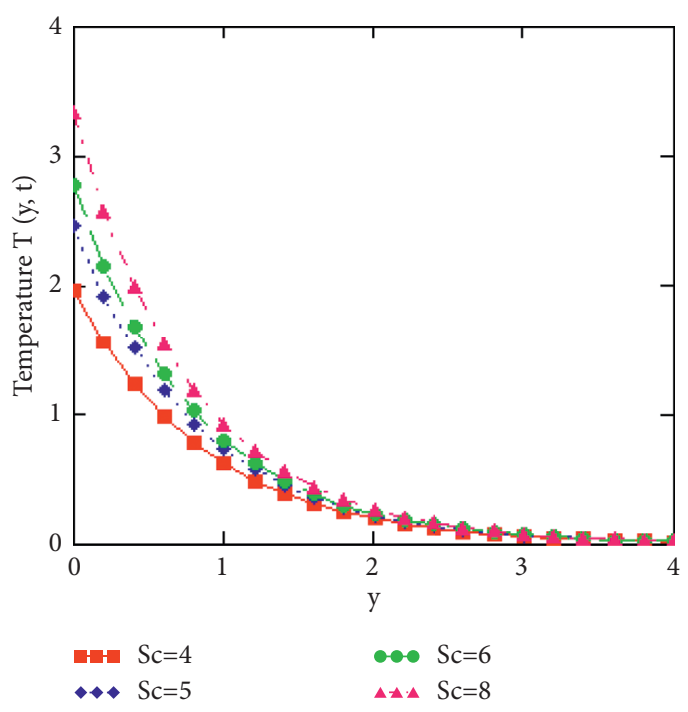

(d)

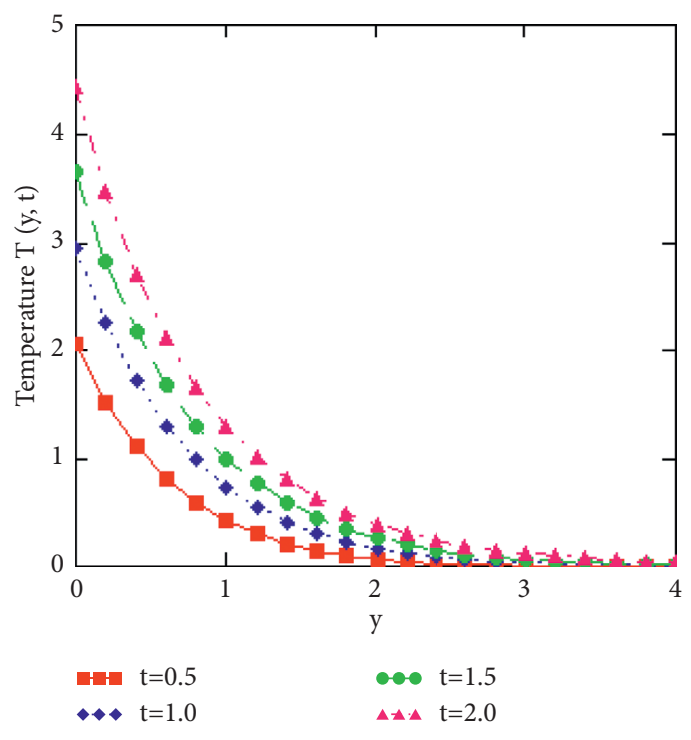

(f)

Figure 3: Temperature profiles vs. for y at $\alpha=0.3, \beta=0.3, \operatorname{Pr}=0.6, S=3, \mathrm{Sc}=4, \mathrm{Du}=0.05, t=2$. 

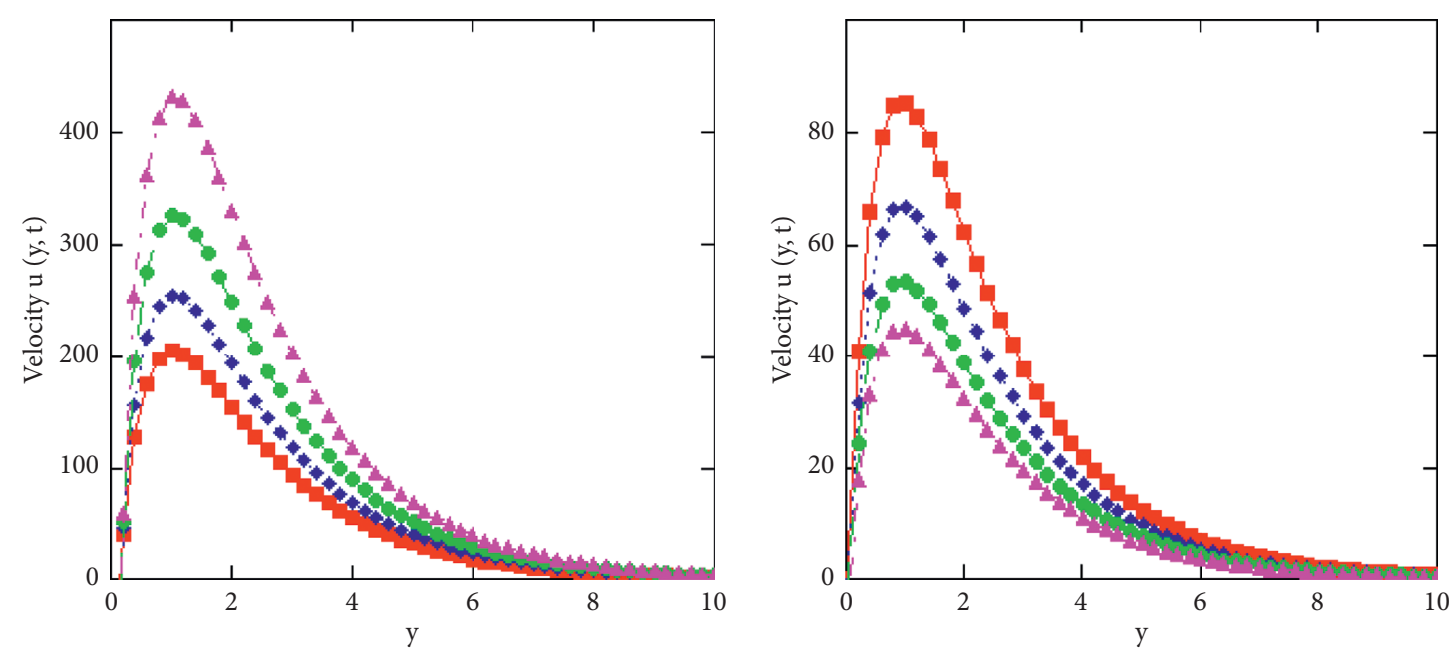

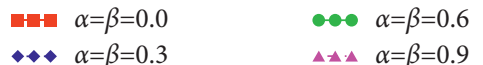

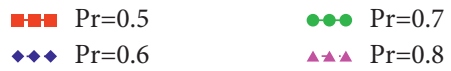

(a)

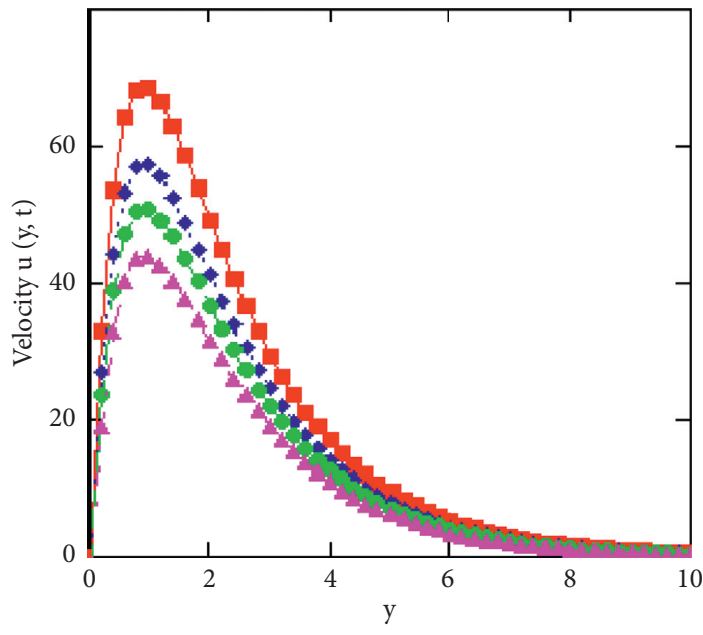

(b)

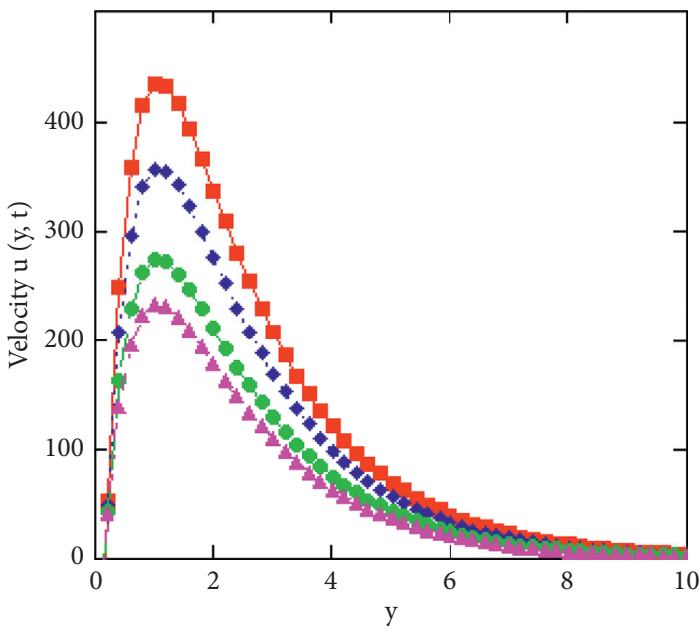

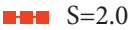

$\leftrightarrow S=3.0$

$\leftrightarrow \mathrm{S}=2.5$

$\Delta \rightarrow A S=3.5$

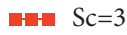

$\cdots \quad \mathrm{Sc}=6$

$\leftrightarrow \mathrm{Sc}=4$

$\Delta \rightarrow \cdot \Delta \mathrm{SC}=8$

(c)

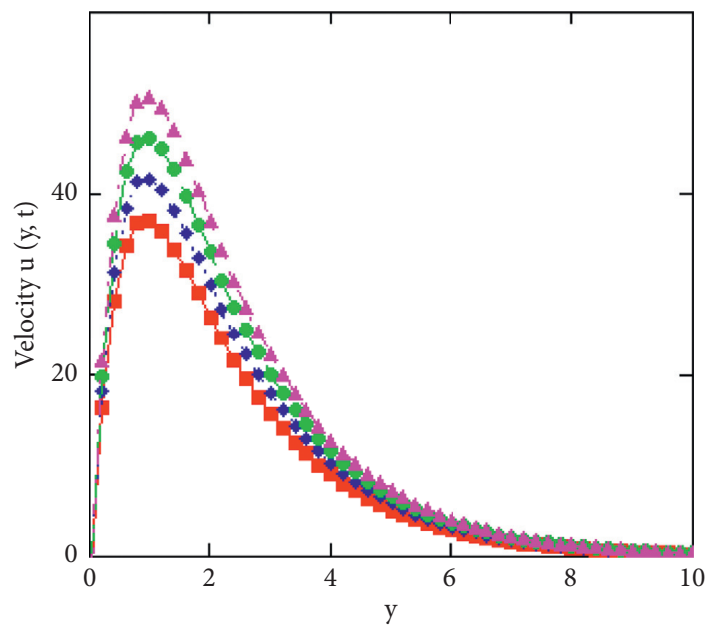

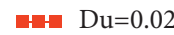

$\leadsto \mathrm{Du}=0.06$

AA.A $\mathrm{Du}=0.08$

(d)

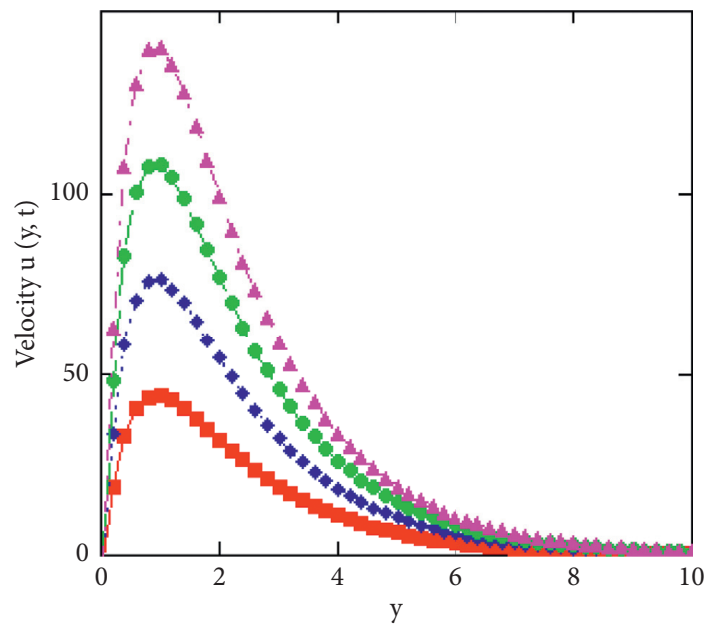

대매매 $\mathrm{Gm}=1.0$

$\ldots \mathrm{Gm}=3.0$

๑ $\mathrm{Du}=0.04$

(e)

(f)

FIgUre 4: Continued. 

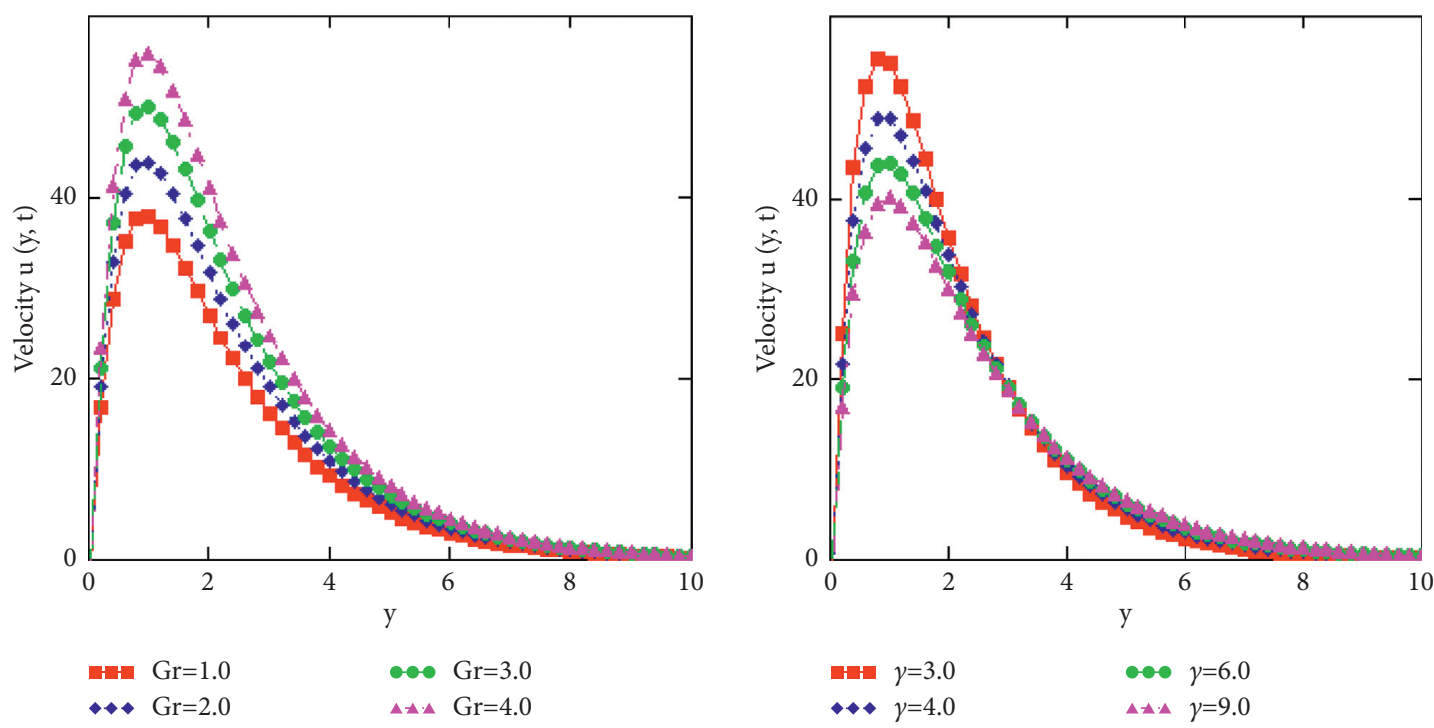

(g)
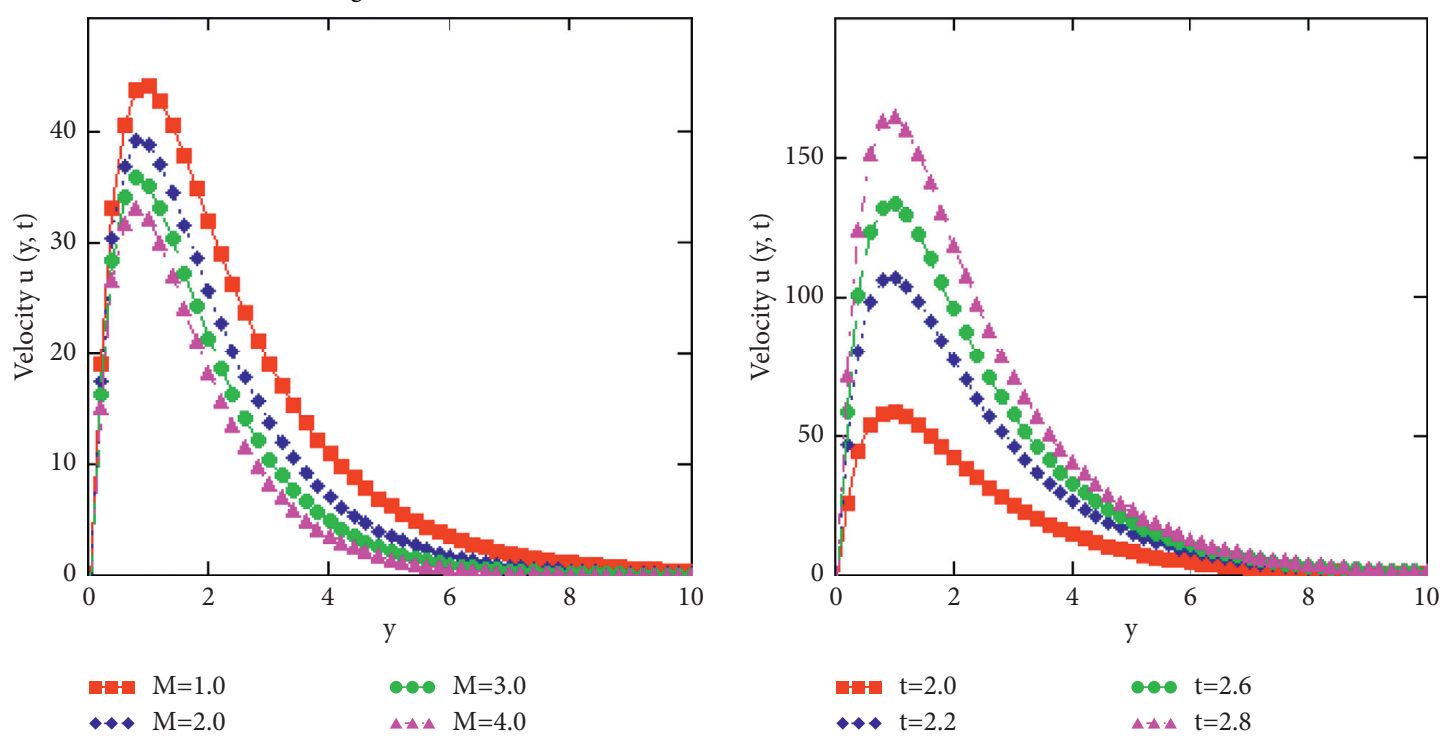

(i)

(j)

Figure 4: Velocity profiles $v s$ for $\mathrm{y}$ at $\alpha=0.3, \beta=0.3, \operatorname{Pr}=0.6, S=3, \mathrm{Sc}=4, \mathrm{Du}=0.05 \mathrm{Du}=0.05, \mathrm{Gm}=1, \mathrm{Gr}=2, \gamma=5, M=1, t=2$.

The impact of fractional parameters $\alpha$ and $\beta$ is described in Table 1. It is attained that the concentration, rate of mass, temperature, rate of heat transfer, velocity, and skin friction are decreasing for the large variation of fractional parameters $\alpha$ and $\beta$.

Furthermore, we have drawn a comparison between fractional second-grade and fractional viscous fluids ordinary fluid models in Figure 5. It is investigated that ordinary fluids have higher velocities as compare to the fractional fluids. It reveals in what way noninteger-order fractional parameters influence the flow of fluid.

Furthermore, to see the validity of our results for concentration, temperature, and velocity profiles graphically, we plotted Figures 6(a)-6(c). It can be seen from these figures that by ignoring the effects of $\mathrm{Du}$ and $M$, our results are identical to those obtained by Vieru et al. [40], Imran et al. [25], and Siddique et al. [33] for fractional fluids. 
TABLE 1: Validation of the model for various values of fractional parameters.

\begin{tabular}{lccccc}
\hline $\begin{array}{l}\text { Fractional parameters } \alpha \text { and } \\
\beta\end{array}$ & $\begin{array}{c}\text { Concentration } \\
(\alpha)\end{array}$ & $\begin{array}{c}\text { Sherwood } \\
\text { number }\end{array}$ & $\begin{array}{c}\text { Temperature }(\alpha \text { and } \\
\beta)\end{array}$ & $\begin{array}{c}\text { Nusselt } \\
\text { number }\end{array}$ & $\begin{array}{c}\text { Velocity } \\
(\alpha \text { and } \beta)\end{array}$ \\
$\begin{array}{l}\text { Skin } \\
\text { friction }\end{array}$ \\
0.0 & 1.000 & 3.950 & 4.444 & 2.459 & 45.583 \\
0.1 & 0.753 & 1.142 & 3.468 & 2.414 & 1.196 \\
0.2 & 0.567 & 0.757 & 2.706 & 2.307 & 155.188 \\
0.3 & 0.426 & 0.570 & 2.112 & 2.153 & 215.824 \\
0.4 & 0.321 & 0.453 & 1.648 & 1.967 & 245.001 \\
0.5 & 0.241 & 0.371 & 1.285 & 1.761 & 251.274 \\
0.6 & 0.181 & 0.310 & 1.002 & 1.548 & 0.646 \\
0.7 & 0.135 & 0.263 & 0.782 & 1.341 & 240.903 \\
0.8 & 0.101 & 0.225 & 0.609 & 1.001 & 226.490 \\
0.9 & 0.075 & 0.194 & 0.475 & 0.337 & 0.1216 \\
\hline
\end{tabular}

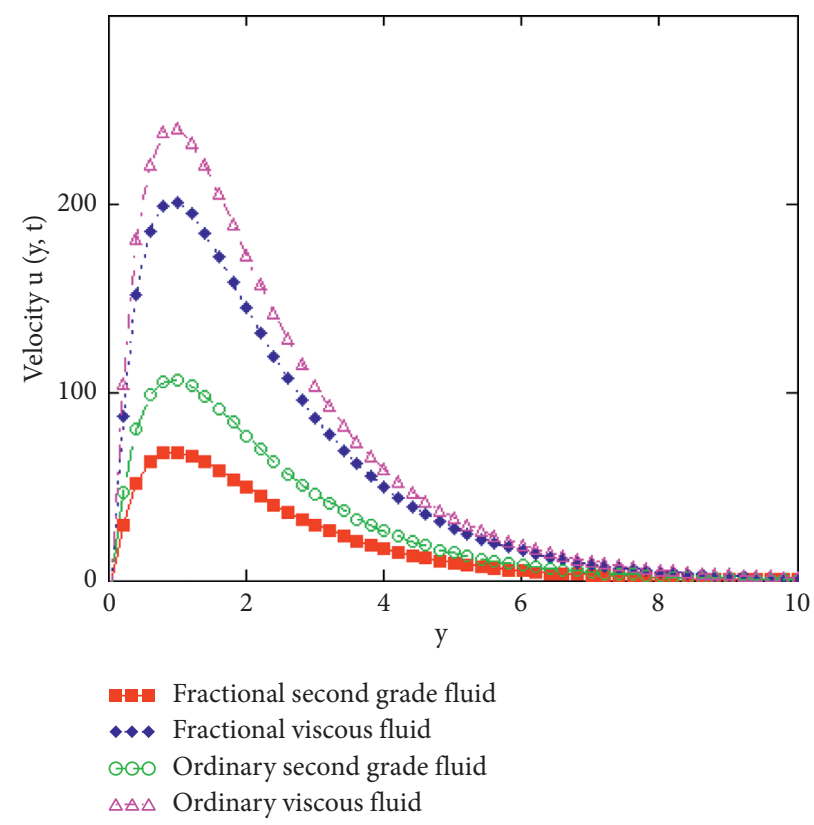

FIGURE 5: Comparison between ordinary and fractional fluids.

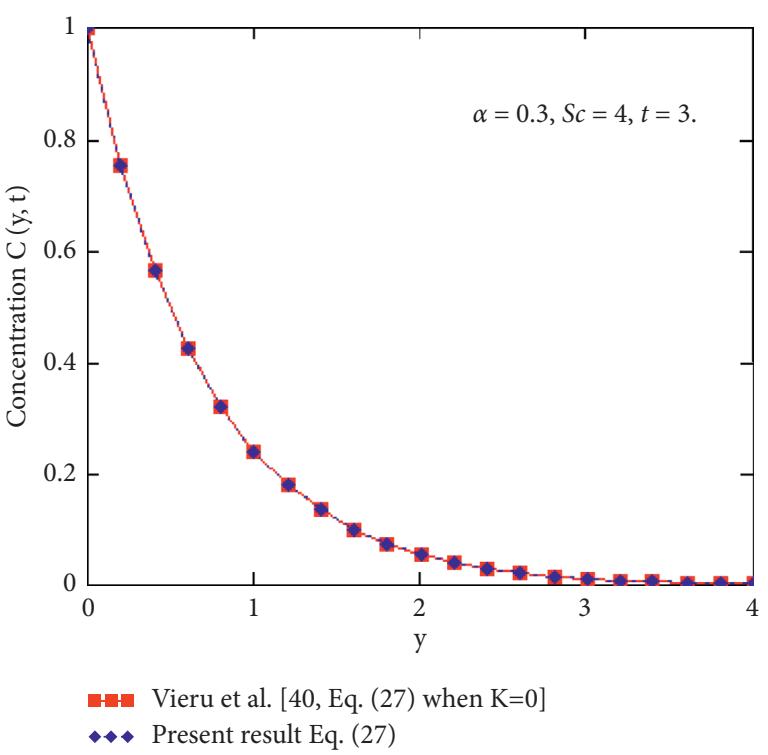

(a)

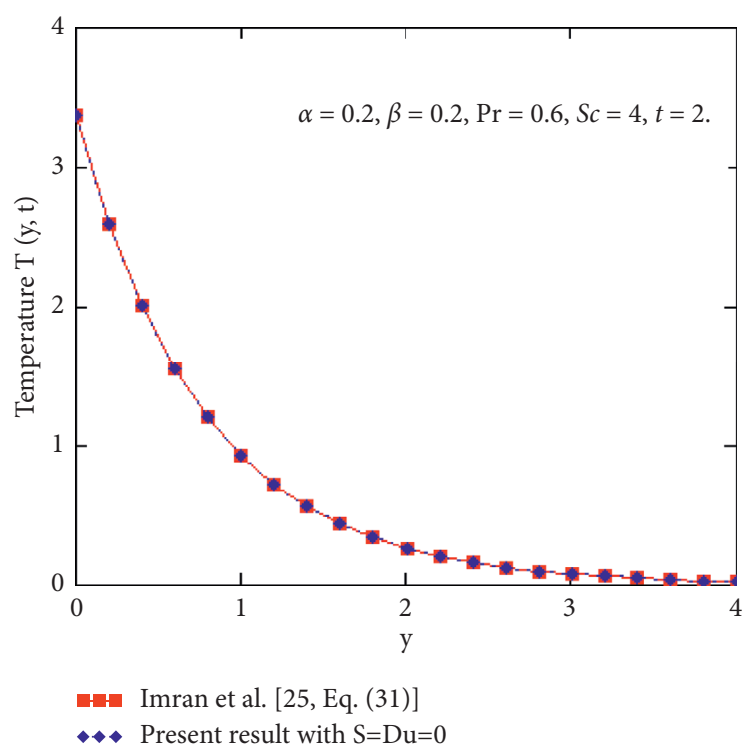

(b)

Figure 6: Continued. 


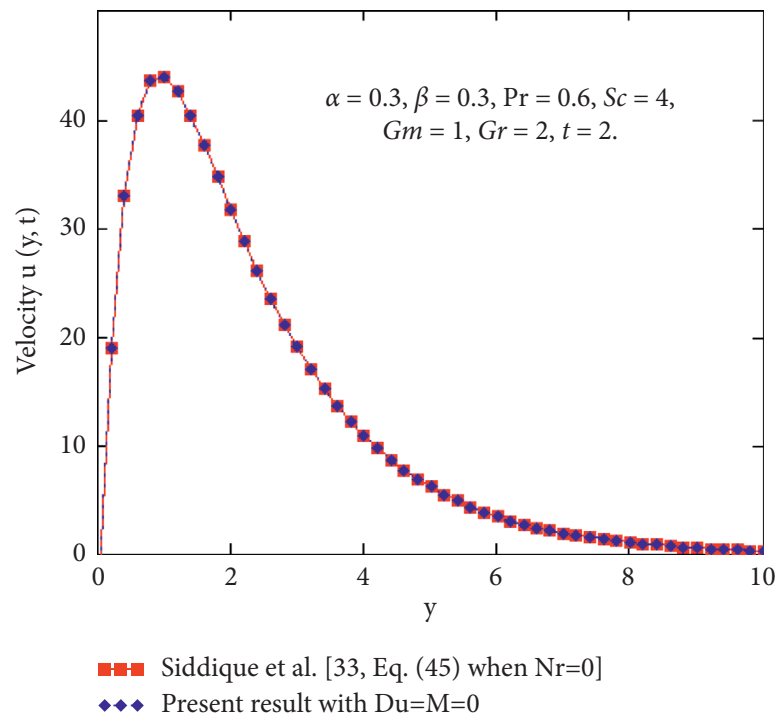

(c)

FiguRE 6: Comparison between the present results with the concentration, temperature, and velocity profiles of Vieru et al. [40], Imran et al. [25], and Siddique et al. [33], respectively.

\section{Conclusion}

In this paper, we analyzed the double convective flow of an incompressible differential-type fluid near a vertical plate with heat absorption, Newtonian heating, and diffusionthermo effect. Time-fractional derivative CF is used in the constitutive equations of the mass flux and thermal flux to describe the diffusion and thermal processes, respectively. Semianalytical solutions of the dimensionless problems are obtained by virtue of the Laplace inversion numerical algorithm Stehfest's. The computations and discussion graphically and numerically have formed to distinguish the effect of CF time-fractional parameters and the second-grade parameter $\gamma$. From numerical simulation and graphical interpretation, the findings are summarized as follows:

(i) For greater values of fractional parameter $\alpha$ and flow parameter $\mathrm{Sc}$, the concentration profile decreases, whereas it increases due to the increasing values of time $t$

(ii) For larger values of fractional parameters $\alpha$ and $\beta$ and flow parameters $\operatorname{Pr}$ and $S$, the temperature profile decreases, whereas it increases due to increasing values of the Dufour number $\mathrm{Du}, \mathrm{Sc}$, and time $t$

(iii) Fluid velocity increases with the increasing values of fractional and flow parameters $\alpha, \beta, \mathrm{Du}, \mathrm{Gm}, \mathrm{Gr}$, and time $t$, and it is observed that the boundary layer thickness increases and velocity is maximum near the plate

(iv) Velocity field as well as the boundary layer thickness decreases near the plate as we increase the values of flow parameters $\operatorname{Pr}, S, \mathrm{Sc}, M$, and $\gamma$

(v) Ordinary fluids (Newtonian and second grade) have greater velocities than fractional fluids (vi) Skin friction, Nusselt numbers, and Sherwood numbers decrease by increasing the fractional parameters $\alpha$ and $\beta$

(vii) The solutions obtained by Vieru et al. [40], Imran et al. [25], and Siddique et al. [33] for fractional fluids are the particular case of our general results for fractional second-grade fluid when $\mathrm{Du}=M=0$, and they are in good agreement graphically

\section{Appendix}

\section{A. Nomenclature}

$\widetilde{u}_{1}$ : velocity field of fluid in the $x$ direction

$\widetilde{\xi}_{1}$ : coordinate axis normal to the plate

$y$ : dimensionless coordinate axis normal to the plate

$\tau_{1}$ : time

$\bar{C}_{\infty}$ : concentration of the fluid far away from the plate

$\widehat{T}_{1}$ : temperature of the fluid near the plate

$T_{\infty}$ : temperature of the fluid far away from the plate

$C_{P}$ : specific heat at a constant pressure

j: mass flux diffusion

$S$ : dimensionless heat absorption parameter

$k_{1}$ : thermal conductivity of the fluid

$D_{m}$ : coefficient of mass diffusivity

Gr: thermal Grashof number

$M$ : magnetic field parameter

Sc: Schmidt number

$u$ : dimensionless velocity

$\bar{C}_{1}$ : species concentration

$\bar{C}_{w}$ : concentration of the plate 
$t$ : dimensionless time

$C$ : dimensionless concentration

$\theta$ : dimensionless temperature

$B_{0}$ : magnetic field parameter

$\bar{q}$ : heat flux

Q: heat absorption parameter

$h_{1}$ : heat transfer coefficient

$C_{s}$ : concentration susceptibility

$K_{T}$ : thermal diffusion ratio

Gm: mass Grashof number

Pr: Prandtl number

$\mathrm{Du}$ : Dufour parameter

Greek symbols

$v$ : kinematic viscosity

$g$ : acceleration due to gravity

$\theta$ : dimensionless temperature

$\beta_{T}$ : volumetric coefficient of thermal expansion

$\beta_{C}$ : volumetric coefficient of expansion with concentration

$\mu$ : dynamic viscosity

$\rho$ : fluid density

$\sigma$ : electrical conductivity

$\alpha_{1}$ : second-grade fluid parameter

$\gamma$ : second-grade coefficient

\section{B. Nondimensional Quantities}

$$
\begin{aligned}
& y=\frac{h_{1} \bar{\xi}_{1},}{k_{1}} \\
& t=v\left(\frac{h_{1}}{k_{1}}\right)^{2} \bar{t}_{1},
\end{aligned}
$$

$$
\begin{aligned}
& u=\frac{1}{v}\left(\frac{k_{1}}{h_{1}}\right) \bar{u}_{1}, \\
& \theta=\frac{\widehat{T}_{1}-\widehat{T}_{\infty}}{\widehat{T}_{\infty}}, \\
& C=\frac{\bar{C}_{1}-\bar{C}_{\infty}}{\bar{C}_{w}-\bar{C}_{\infty}}, \\
& q=\frac{\bar{q}}{h_{1} \widetilde{T}_{\infty}}, \\
& j=\frac{\widehat{j} k_{1}}{\nu\left(\bar{C}_{w}-\widehat{C}_{\infty}\right) h_{1}}, \\
& \operatorname{Pr}=\frac{\mu C_{p}}{k_{1}}, \\
& \mathrm{Sc}=\frac{v}{D_{m}}, \\
& \gamma=\frac{h_{1}^{2} \alpha_{1}}{\rho k_{1}^{2}}, \\
& \mathrm{Gr}=\frac{g \beta_{T} k_{1}^{3} \widehat{T}_{\infty}}{v^{2} h_{1}^{3}}, \\
& \mathrm{Gm}=\frac{g \beta_{C} k_{1}^{3}\left(\bar{C}_{w}-\widehat{C}_{\infty}\right)}{\nu^{2} h_{1}^{3}}, \\
& S=\frac{Q}{k_{1}}\left(\frac{k_{1}}{h_{1}}\right)^{2} \text {, } \\
& \mathrm{Du}=\frac{D_{m} K_{T}\left(\bar{C}_{w}-\bar{C}_{\infty}\right)}{C_{s} C_{p} \widehat{T}_{\infty}}, \\
& M=\frac{\sigma B_{0}^{2} k_{1}^{2}}{\mu h_{1}^{2}} \text {. }
\end{aligned}
$$




\section{Some Constants Involved in the Text}

$$
\begin{aligned}
& a_{1}=\frac{S}{\operatorname{Pr}}, \\
& a_{2}=-\frac{\mathrm{PrDu}}{(\mathrm{Sc}-\mathrm{Pr})}, \\
& a_{3}=-\frac{S}{\mathrm{Sc}-\mathrm{Pr}} \text {, } \\
& a_{4}=\frac{a_{0}^{2}(1+a \gamma)-1}{a_{0}^{2} \gamma}, \\
& a_{5}=\frac{a_{0}^{2} a-M}{a_{0}^{2} \gamma}, \\
& b_{0}=(1-\beta) \operatorname{Pr}, \\
& b_{1}=\frac{S+b \operatorname{Pr}}{\operatorname{Pr}}, \\
& b_{2}=\frac{b S}{\operatorname{Pr}}, \\
& b_{3}=-\frac{\operatorname{PrDu} a_{0}^{2}(1-\beta)}{\operatorname{Sc}(1-\alpha)}, \\
& b_{4}=\frac{a_{0}^{2} a-b_{0} b_{1}}{a_{0}^{2}-b_{0}}, \\
& b_{5}=-\frac{b_{0} b_{2}}{a_{0}^{2}-b_{0}}, \\
& b_{6}=\frac{b_{3}}{a_{0}^{2}-b_{0}}, \\
& d_{0}=\frac{b_{0}+b_{0} b_{1}-1}{b_{0} \gamma}, \\
& d_{1}=\frac{b_{0} b_{1}+b_{0} b_{2} \gamma-M}{b_{0} \gamma}, \\
& d_{2}=\frac{b_{2}}{\gamma}, \\
& d_{3}=\frac{1}{\gamma} \text {, } \\
& p_{1}=\frac{b_{0} b_{1}-M}{b_{0}-1}, \\
& p_{2}=\frac{b_{0} b_{2}}{b_{0}-1} \\
& p_{3}=\frac{a a_{0}^{2}-M}{a_{0}^{2}-1}, \\
& p_{4}=\frac{(\operatorname{Pr})^{3 / 2}\left(1+a_{1} \gamma\right)-1}{(\operatorname{Pr})^{3 / 2} \gamma}, \\
& p_{5}=\frac{a_{1}(\operatorname{Pr})^{3 / 2}-M}{(\operatorname{Pr})^{3 / 2} \gamma}, \\
& p_{6}=\frac{\operatorname{Pr} a_{1}-M}{\operatorname{Pr}-1} \text {, } \\
& p_{7}=-\frac{M}{\operatorname{Pr}-1} \text {, } \\
& A_{1}=\frac{\mathrm{Gr}}{b_{0}^{3 / 2} \gamma}, \\
& A_{2}=A_{1} b_{6} \text {, } \\
& A_{3}=\frac{\mathrm{Gr} b_{6}}{a_{0}^{2} \gamma} \text {, } \\
& A_{4}=\frac{\mathrm{Gm}}{a_{0}^{2} \gamma} \text {, } \\
& A_{5}=\frac{\mathrm{Gr}}{\sqrt{b_{0}}\left(b_{0}-1\right)}, \\
& A_{6}=A_{1} b_{6} \text {, } \\
& A_{7}=\frac{\mathrm{Gr} b_{6}}{a_{0}^{2}-1} \text {, } \\
& A_{8}=\frac{\mathrm{Gm}}{a_{0}^{2}-1} \text {, } \\
& A_{9}=\frac{\mathrm{Gr}}{(\mathrm{Pr})^{3 / 2} \gamma} \text {, } \\
& A_{10}=\frac{\mathrm{Gr} a_{2}}{\mathrm{Sc} \gamma}, \\
& A_{11}=\frac{\mathrm{Gm}}{\mathrm{Sc} \gamma} \text {, } \\
& A_{12}=A_{13}=\frac{\mathrm{Gr}}{\mathrm{Sc}-1} \text {, } \\
& A_{14}=\frac{\mathrm{Gm}}{\mathrm{Sc}-1} \text {, } \\
& \xi=-\frac{1}{\sqrt{b_{0}}}, \\
& \zeta=-\frac{1}{\sqrt{\operatorname{Pr}}} \text {. }
\end{aligned}
$$




\section{Some Inverse Laplace Formulas}

$$
\begin{aligned}
& L^{-1}\left\{\frac{e^{-a \sqrt{s+b}}}{s}\right\}=\frac{1}{2}\left[e^{a \sqrt{b}} \operatorname{erfc}\left(\frac{a}{2 \sqrt{t}}+\sqrt{b t}\right)+e^{-a \sqrt{b}} \operatorname{erfc}\left(\frac{a}{2 \sqrt{t}}-\sqrt{b t}\right)\right] \\
& L^{-1}\left\{\frac{e^{-a \sqrt{s}}}{s}\right\}=\operatorname{erfc}\left(\frac{a}{2 \sqrt{t}}\right) \\
& \text { If } g_{1}(t)=L^{-1}\left\{G_{1}(s)\right\} \text {, then } L^{-1}\left\{G_{1}(\psi(s))\right\}=\int_{0}^{\infty} g_{1}(r) h_{1}(r, t) \mathrm{d} r \text {, } \\
& \text { where } h_{1}(r, t)=L^{-1}\{\exp (-r \psi(s))\} \text {, } \\
& L^{-1}\left\{e^{-(a /(s+b))}\right\}=e^{-b t}\left\{\delta(t)-\sqrt{\frac{a}{t}} J_{1}(2 \sqrt{a t})\right\}, \\
& L^{-1}\left\{\frac{1}{s^{2}+b_{1} s+b_{2}}\right\}=\frac{2}{\sqrt{b_{1}^{2}-4 b_{2}}} \sinh \left(\frac{\sqrt{b_{1}^{2}-4 b_{2}}}{2} t\right) e^{-\left(b_{1} / 2\right) t}, \\
& L^{-1}\{\sqrt{s+a}\}=\frac{e^{-a t}}{2 t \sqrt{\pi t}} \\
& L^{-1}\left\{\frac{1}{\sqrt{s+a}+b}\right\}=e^{-a t}\left\{\frac{1}{\sqrt{\pi t}}-a_{0} e^{b^{2} t} \operatorname{erf} c(b \sqrt{t})\right\} \\
& L^{-1}\left\{\frac{e^{-a \sqrt{s+c}}}{\sqrt{s+c}+b}\right\}=e^{-c t}\left\{\frac{e^{-a^{2} / 4 t}}{\sqrt{\pi t}}-b e^{a b+b^{2} t} \operatorname{erf} c\left(\frac{a}{2 \sqrt{t}}+b \sqrt{t}\right)\right\}, \\
& \int_{0}^{t} \xi^{-3 / 2} \exp \left(u^{2} \xi-\frac{x^{2}}{\xi}\right) \mathrm{d} \xi=\frac{\sqrt{\pi}}{2 x}\left[e^{-2 i u x} \operatorname{erf} c\left(\frac{x}{\sqrt{t}}-i u \sqrt{t}\right)+e^{2 i u x} \operatorname{erf} c\left(\frac{x}{\sqrt{t}}+i u \sqrt{t}\right)\right], \\
& L^{-1}\left\{\frac{1}{s(\sqrt{s+b}+c)}\right\}=\frac{1}{b-c^{2}}\left\{\sqrt{b} \operatorname{erf}(b \sqrt{t})-\left(e^{-\left(b-c^{2}\right)}\right) \operatorname{erf} c(c \sqrt{t})-1\right\} \\
& L^{-1}\left\{\frac{e^{-y \sqrt{(s+b) /(s+c)}}}{s}\right\}=e^{-y}-\frac{y \sqrt{b-c}}{2 \sqrt{\pi}} \int_{0}^{\infty} \int_{0}^{t} \frac{1}{\sqrt{t}} e^{-\left(c t+\left(y^{2} / 4 u\right)+u\right)} I_{1}(2 \sqrt{(b-c) u t}) \mathrm{d} t \mathrm{~d} u .
\end{aligned}
$$

\section{Data Availability}

No data were used to support this study.

\section{Conflicts of Interest}

The authors declare that they have no conflicts of interest.

\section{References}

[1] Y. Jaluria, Natural Convection Heat and Mass Transfer, Pergamon Pergamon Press, Oxford, UK, 1980.
[2] P. S. Ghoshdastidar, Heat Transfer, Oxford University Press, Oxford, UK, 2004.

[3] M. A. Imran, I. Khan, M. Ahmad, N. A. Shah, and M. Nazar, "Heat and mass transport of differential type fluid with noninteger order time-fractional Caputo derivatives," Journal of Molecular Liquids, vol. 229, pp. 67-75, 2017.

[4] C. Fetecau, D. Vieru, C. Fetecau, and I. Pop, "Slip effects on the unsteady radiative MHD free convection flow over a moving plate with mass diffusion and heat source," The European Physical Journal Plus, vol. 130, no. 1, p. 6, 2015.

[5] B. R. Sharma and K. Nath, "Effects of magnetic field and variation of viscosity and thermal conductivity on separation of a binary fluid mixture over a continuously moving surface," 
International Journal for Innovative Research in Science, Engineering and Technology, vol. 2, no. 11, pp. 6516-6524, 2013.

[6] S. Parvin and R. Nasrin, "Analysis of the flow and heat transfer characteristics for MHD free convection in an enclosure with a heated obstacle," Nonlinear Analysis: Modelling and Control, vol. 16, no. 1, pp. 89-99, 2011.

[7] E. R. G. Eckert and R. M. Drake, Analysis of Heat and Mass Transfer, McGraw-Hill Book Co., New York, NY, USA, 1972.

[8] N. G. Kafoussias and E. W. Williams, "Thermal-diffusion and diffusion-thermo effects on mixed free-forced convective and mass transfer boundary layer flow with temperature dependent viscosity," International Journal of Engineering Science, vol. 33, no. 9, pp. 1369-1384, 1995.

[9] K. R. Babu, A. G. V. Kumar, and S. V. K. Varma, "Diffusionthermo and radiation effects on MHD free convective heat and mass transfer flow past an infinite vertical plate in the presence of a chemical reaction of first order," Advances in Applied Science Research, vol. 3, no. 4, pp. 2446-2462, 2012.

[10] U. S. Rajput and N. K. Gupta, "Dufour effect on unsteady free convection MHD flow past an exponentially accelerated plate through porous media with variable temperature and constant mass diffusion in an inclined magnetic field," International Research Journal of Engineering and Technology, vol. 3, no. 8, pp. 2135-2140, 2016.

[11] B. R. Sharma and B. Buragohain, "Soret and Dufour effects on unsteady flow past an oscillating vertical plate," International Journal for Innovative Research in Science, Engineering and Technology, vol. 2, no. 8, pp. 2349-6010, 2016.

[12] A. Postelnicu, "Influence of a magnetic field on heat and mass transfer by natural convection from vertical surfaces in porous media considering Soret and Dufour effects," International Journal of Heat and Mass Transfer, vol. 47, no. 6-7, pp. 1467-1472, 2004.

[13] S. N. Gaikwad, M. S. Malashetty, and K. Rama Prasad, "An analytical study of linear and non-linear double diffusive convection with Soret and Dufour effects in couple stress fluid," International Journal of Non-linear Mechanics, vol. 42, no. 7, pp. 903-913, 2007.

[14] J. Prakash, D. Bhanumathi, A. G. Vijaya Kumar, and S. V. K. Varma, "Diffusion-thermo and radiation effects on unsteady MHD flow through porous medium past an impulsively started infinite vertical plate with variable temperature and mass diffusion," Transport in Porous Media, vol. 96, no. 1, pp. 135-151, 2013.

[15] R. S. Rivlin, "The hydrodynamics of non-Newtonian fluids-I," Proceedings of the Royal Society of London A, vol. 193, pp. 260-281, 1948.

[16] R. S. Rivlin and J. L. Ericksen, "Stress deformation relations for isotropic materials," Journal of Rational Mechanics and Analysis, vol. 4, no. 21, pp. 323-425, 1955.

[17] D. W. Beard and K. Walters, "Elastico-viscous boundary-layer flows I. Two-dimensional flow near a stagnation point," Mathematical Proceedings of the Cambridge Philosophical Society, vol. 60, no. 3, pp. 667-674, 1964.

[18] P. Donald Ariel, "On exact solutions of flow problems of a second grade fluid through two Parallel porous walls," International Journal of Engineering Science, vol. 40, pp. 913941, 2002.

[19] A. Kecebas and M. Yurusoy, "Numerical solutions of unsteady boundary layer equations for a generalized second grade fluid," Journal of Theoretical and Applied Mechanics, vol. 49, no. 1, pp. 71-82, 2011.
[20] B. Raftari, F. Parvaneh, and K. Vajravelu, "Homotopy analysis of the magnetohydrodynamic flow and heat transfer of a second grade fluid in a porous channel," Energy, vol. 59, no. 15 , pp. $625-632,2013$.

[21] S. Aman, Z. Ismail, M. Z. Salleh, and I. Khan, "Flow analysis of second grade fluid with wall suction/injection and convective boundary condition," Journal of Advanced Research in Fluid Mechanics and Thermal Sciences, vol. 58, no. 1, pp. 135-143, 2019.

[22] R. Hilfer, Threefold Introduction to Fractional Derivatives, Anomalous Transport: Foundations and Applications,, Stuttgart, Germany, 2008.

[23] R. Gorenflo, F. Mainardi, D. Moretti, and P. Paradisi, "Time fractional diffusion: a discret random walk approach," Nonlinear Dynamics, vol. 29, pp. 129-143, 2002.

[24] M. Caputo and M. Fabrizio, "A new definition of fractional derivative without singular kernel," Progress in Fractional Differentiation and Applications, vol. 1, no. 2, pp. 73-85, 2015.

[25] M. I. Asjad, N. A. Shah, M. Aleem, and I. Khan, "Heat transfer analysis of fractional second-grade fluid subject to Newtonian heating with Caputo and Caputo-Fabrizio fractional derivatives: a comparison," The European Physical Journal Plus, vol. 132, p. 340, 2017.

[26] S. Akhter, "Flows between two parallel plates of Couple stress fluids with time-fractional Caputo and Caputo-Fabrizio derivative," The European Physical Journal Plus, vol. 131, p. 401, 2016.

[27] S. Ullah, S. Zulfiqar, A. A. Buhader, and N. A. Khan, “Analysis of Caputo-Fabrizio fractional order semi-linear parabolic equations via effective amalgamated technique," Physica Scripta, vol. 96, no. 3, p. 035214, 2021.

[28] M. Ali Dokuyucu, "A fractional order alcoholism model via Caputo-Fabrizio derivative,” AIMS Mathematics, vol. 5, no. 2, pp. 781-797, 2020.

[29] M. Saqib, I. Khan, S. Shafie, and A. Q. Mohamad, "Shape effect on MHD flow of time fractional Ferro-Brinkman type nanofluid with ramped heating," Scientific Reports, vol. 11, p. $3725,2021$.

[30] M. Aleem, M. I. Asjad, A. Shaheen, and I. Khan, "MHD influence on different water based nanofluids $\left(\mathrm{TiO}_{2}, \mathrm{Al}_{2} \mathrm{O}_{3}\right.$, $\mathrm{CuO}$ ) in porous medium with chemical reaction and Newtonian heating," Chaos, Solitons \& Fractals, vol. 130, p. 109437, 2020.

[31] M. B. Riaz and N. Iftikhar, "A comparative study of heat transfer analysis of MHD Maxwell fluid in view of local and nonlocal differential operators," Chaos, Solitons \& Fractals, vol. 132, p. 109556, 2020.

[32] S. Ul Haq, S. Ullah Jan, S. Inayat Ali Shah, I. Khan, and J. Singh, "Heat and mass transfer of fractional second grade fluid with slippage and ramped wall temperature using Caputo-Fabrizio fractional derivative approach," AIMS Mathematics, vol. 5, no. 4, pp. 3056-3088, 2020.

[33] I. Siddique and S. M. Bukhari, "Analysis of the effect of generalized fractional Fourier's and Fick's laws on convective flows of non-Newtonian fluid subject to Newtonian heating," The European Physical Journal Plus, vol. 135, no. 1, p. 45, 2020.

[34] I. Siddique, I. Tlili, S. M. Bukhari, and Y. Mahsud, "Heat transfer analysis in convective flows of fractional second grade fluids with Caputo-Fabrizio and Atangana--Baleanu derivative subject to Newtonion heating," Mechanics of Time-Dependent Materials, 2020.

[35] H. Stehfest, "Algorithm 368: numerical inversion of Laplace transforms [D5]," Communications of the ACM, vol. 13, no. 1, pp. 47-49, 1970. 
[36] K. L. Kuhlman, "Review of inverse Laplace transform algorithms for Laplace-space numerical approaches," Numerical Algorithms, vol. 63, no. 2, pp. 339-355, 2013.

[37] B. C. Tai and M. I. Char, "Soret and Dufour effects on free convection flow of non-Newtonian fluids along a vertical plate embedded in a porous medium with thermal radiation," International Communications in Heat and Mass Transfer, vol. 37, no. 5, pp. 480-483, 2010.

[38] J. Hristove, Fronttiers in Fractional Calculus, S. Bhalekar, Ed., pp. 235-295, Bentham Science Publishers, Sharjah, UAE, 1st edition, 2017.

[39] Y. Povstenko, "Fractional thermoelasticity," in Encyclopedia of Thermal Stresses, R. B. Hetnarski, Ed., vol. 4, pp. 1778-1787, Springer, New York, NY, USA, 2014.

[40] D. Vieru, C. Fetecau, and C. Fetecau, "Time fractional free convection flow near a vertical plate with Newtonian heating and Mass diffusion," Thermal Science, vol. 19, no. 1, pp. S85-S98, 2015. 